\title{
Fast 3D flow reconstructions from 2D cross-plane observations
}

\author{
Pranav Chandramouli ${ }^{1}$, Etienne Memim $^{1}$, Dominique Heitz ${ }^{1,2}$, Lionel Fiabane ${ }^{2}$ \\ 1. INRIA, Fluminance group, Campus universitaire de Beaulieu, \\ F-35042 Rennes Cedex, France \\ 2. Irstea, UR OPAALE, F-35044 Rennes Cedex, France
}

February 8, 2019

\begin{abstract}
A computationally efficient flow reconstruction technique is proposed, exploiting homogeneity in a given direction, to recreate three dimensional instantaneous turbulent velocity fields from snapshots of two dimension planar fields. This methodology, termed as 'snapshot optimisation' or SO, can help provide 3D data-sets for studies which are currently restricted by the limitations of experimental measurement techniques. The SO method aims at optimising the error between an inlet plane with a homogeneous direction and snapshots, obtained over a sufficient period of time, on the observation plane. The observations are carried out on a plane perpendicular to the inlet plane with a shared edge normal to the homogeneity direction. The method is applicable to all flows which display a direction of homogeneity such as cylinder wake flows, channel flow, mixing layer, and jet (axi-symmetric). The ability of the method is assessed with two synthetic data-sets, and three experimental PIV data-sets. A good reconstruction of the large-scale structures are observed for all cases. The small-scale reconstruction ability is partially limited especially for higher-dimensional observation systems. POD based SO method and averaging SO variations of the method are shown to reduce discontinuities created due to temporal mismatch in the homogenous direction providing a smooth velocity reconstruction. The volumetric reconstruction is seen to capture large-scale structures for synthetic and experimental casestudies. The algorithm run-time is found to be in the order of a minute providing results comparable with the reference. Such a reconstruction methodology can provide important information for data assimilation in the form of initial condition, background condition, and $3 \mathrm{D}$ observations.
\end{abstract}

\section{Introduction}

With the advent of particle image velocimetry (PIV) in the 1980's (Adrian, 1984), the field of experimental fluid dynamics grew exponentially with the ability now to measure data over large 2D domains. Further improvements saw the measurement of velocity fields in 3D albeit at limited spatial resolutions using tomographic-PIV (tomo-PIV) techniques (Scarano, 2012) or more recently using 3D+time Particle Tracking Velocimetry (4D-PTV) techniques (Schanz et al. 2016). However, these methods are still quite restricted in terms of their spatial and temporal resolutions as well as the ability to observe the full state-space vectors. To improve 3D spatiotemporal PTV measurements data Navier-Stokes constraints have been proposed in an optimisation framework. Schneiders \& Scarano (2016) use the vortex in cell plus (VIC+) technique to reconstruct instantaneous fields from time-resolved measurements using the velocities and the material derivatives. In a similar approach, Gesemann et al. (2016) proposed FlowFit, a similar approach to interpolate Lagrangian measured velocities to a regular grid. The aim of all these methods has been to reconstruct 3D full-scale observations from sparse partial data-sets. 
An upcoming methodology to complete this partial state-space vector obtained from an experiment is to perform Data Assimilation (DA), wherein a combination of computational methods (CFD) and experimental data (EFD) is used to complete the missing data. However, DA is limited in performance by the numerical methods employed and more importantly, the quality of the experimental data used to guide the numerical simulation.

PIV data-sets provide a good spatial and temporal resolution of data in 2D. Many studies exist in literature that use this data to perform 2D DA to estimate an improved field of interest (see the works of Gronskis et al. (2013), Fujisawa et al. (2005), and D'adamo et al. (2007)). Until recently, the extent of DA studies have been limited to 2D due to inhibitive costs of performing DA - both the variational and the sequential approach to DA are computationally expensive. The variational approach, formulated as an optimal control problem, seeks to minimise the error between the simulation and the observation data by estimating an optimal trajectory bounded by physical laws. Such a minimisation procedure relies on the adjoint formulation of the associated dynamics for calculating the gradient based on the works of Lions (1971), and Le Dimet \& Talagrand (1986). To formulate, implement and use this adjoint formulation can be tedious and numerically difficult. In the sequential approach, based on stochastic filtering techniques, multiple realisations of the state vector are propagated in time taking into account available measurements at each time. Such a formulations suffers from the 'curse of dimensionality' (Yang et al. , 2015) as the few samples considered are unlikely to represent the large state-space. In addition, the ease of adaptability of many sequential approaches to high-dimensional, non-linear problems is questionable (Mons et al., 2016).

In the field of variational DA in fluid dynamics, due to the use of turbulence modelling such as Large Eddy Simulations (LES), and Reynolds-averaged Navier-Stokes (RANS) models, an expansion into three dimensional assimilation studies have recently been achieved (Chandramouli et al., 2017). A DNS based 3D DA study was performed by Robinson (2015) at a very low Reynolds number (Re) of 300 for flow around circular cylinder. As we expand into higher Re flows, the need for 3D data-sets describing the flow becomes imperative. Existing 3D DA studies either rely on non-physical mirror-imaging technique, or interpolation/extrapolation techniques to obtain 3D observation data from existing 2D PIV data or involve complex timeconsuming methodologies such as the 'Empty Box' technique of (Robinson, 2015). Tomo-PIV and PTV techniques are capable of producing 3D flow fields but are still limited in their spatial temporal extent to sparse observations. Thus, a quick and effective method to reconstruct the velocity field over a volume could be very useful for 3D DA studies until advances in experimental techniques become capable of capturing spatially well-resolved observations in a large 3D domain.

Simple methods for 3D complex flow reconstruction from 2D PIV data have been proposed in literature. The scanning PIV technique proposed by Brücker (1995) for the analysis of 3D effects in a cylinder wake flow involves classical PIV technique with the scanning of a volume via a light sheet. Since then this approach has been carried out for single (Zhang et al., 2008; Brücker et al., 2012) or stereo (Casey et al., 2013) camera arrangements. The technique assumes a frozen velocity field during the scanning process that thus need to be sufficiently quick. With the assumption of frozen turbulence via Taylor hypothesis, Ganapathisubramani et al. (2008) used cinematographic stereoscopic PIV to transform time into space and reconstruct a quasi-instantaneous volume of data in a turbulent jet. The PIV plane was perpendicular to the streamwise flow direction. Steinberg et al. (2009) developed an orthogonal-plane cinematographic stereoscopic PIV to study turbulence -flame interactions. The plane perpendicular to the streamwise flow was used for the $3 \mathrm{D}$ reconstruction of the flow and the plane parallel to the streamwise flow direction to observe the interactions between the turbulent structures and the flame. In order to obtain simultaneous spatiotemporal velocity information for 3D flow structure investigation, Kähler \& Kompenhans (2000) studied multiple plane stereo PIV. 
Braud et al. (2004) carried out such an approach in a dual plane stereo PIV configuration combined with 3D POD to reconstruct and analyse the flow structures of the circular cylinder near wake-mixing layer interaction. Kit et al. (2005) proposed another approach coupling hot-wire measurements, to obtain detailed time-series, and a limited number of phase-locked stereo PIV measurements to reconstruct time variations of large coherent structures in a forced mixing layer. More recently in the same spirit, Hamdi et al. (2018) synchronised with a trigger signal parallel planes stereo PIV and POD to reconstruct a volume of an impinging jet. Foucaut et al. (2011) focused on computing the 3D correlation tensor from stereoscopic PIV data by exploiting homogeneity along two principle directions.

In this work, we aim to utilising homogeneity along one principle direction in order to reconstruct the 3D flow field from 2D data-sets obtained on two perpendicular planes, easily available from established experimental methods such as stereo PIV. Towards this, we propose a quick and effective algorithm called the snapshot optimisation (SO) methodology which is formulated in the next section. This is followed by a section analysing the performance of the algorithm using varied sets of observational data - both synthetic and experimental. A final section of concluding remarks follows.

\section{Snapshot optimisation method}

The concept of the SO method and the algorithm inputs are explained in section 2.1. The mathematical formulation of the optimisation problem is provided in section 2.2. A reduced order version of the model using proper orthogonal decomposition (POD) is discussed in section 2.3 . An averaging variant of the model is proposed in section 2.4 .

\subsection{SO model concept}

Consider a flow exhibiting a direction of homogeneity, for example, wake flow around a circular cylinder. Figure 1 depicts the geometry for this flow with the two planes of measurements. The plane in red, parallel to the cylinder axis, is referred to as the inlet plane (IP) while the plane in green, perpendicular to the cylinder, is referred to as the observation plane (OP). While the OP has been depicted in the middle of the domain, this is not a necessary condition of the reconstruction - any plane perpendicular to the spanwise $z$-direction can be utilised as the OP. The objective is to reconstruct the $3 \mathrm{D}$ domain encompassing the IP and the OP, from only one $3 \mathrm{C}$ vector field in the IP and an ensemble of $3 \mathrm{C}$ vectors fields in the OP.

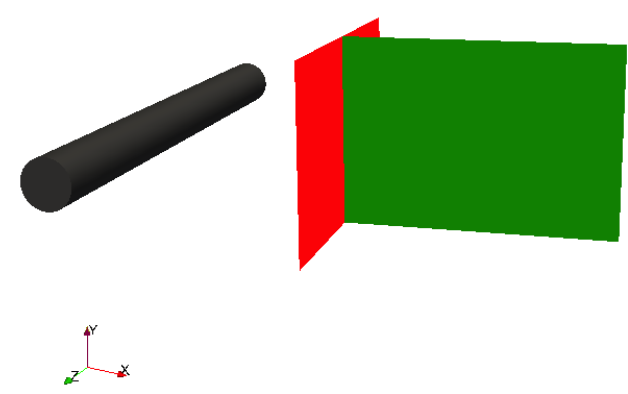

Figure 1: Geometry for wake flow around a circular cylinder with planes of measurement

This 3D domain can be considered to be made up of a set of 2D parallel planes (a sample decomposition containing three such planes is shown in figure 2a). Each parallel plane is assumed to begin at the IP thus sharing a common edge (along $y$ ) with the inlet plane. Now, we make the critical assumption: considering the turbulence homogeneity of the flow along the spanwise $z$-direction, a sufficiently long time-sequence of snapshots of the observation plane 
(in green in figure 1) contains at some point within that time-sequence a representation of each of the 2D parallel planes of figure 2a. This is graphically shown in figure 2b. In other words, it is assumed that one event constituted by one planar velocity field on a given plane, is observed at a given time of the observations sequence. Such a reconstruction is along the lines of the "pouring time in to space" techniques of Schneiders \& Scarano (2016).

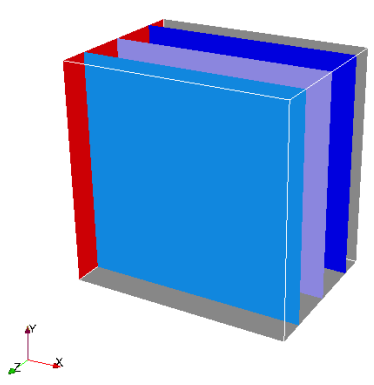

(a) 3D domain split in to multiple parallel $2 \mathrm{D}$ plane observations

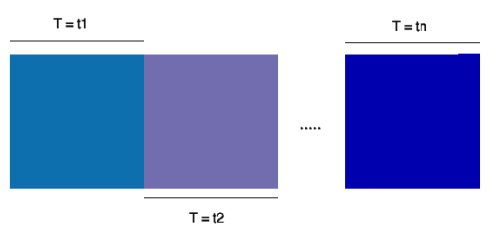

(b) 2D planes represented within the timesequence of the observation plane measurements.

Figure 2: 3D domain of interest shown within the original volume and within the observation measurements

Thus, to reconstruct the 3D domain of interest from the 2D measurements, we need to identify the snapshots within the OP time-sequence which correspond to each of the parallel planes comprising the volume. Since all of these planes share an edge with the inlet plane where measurements are available, an optimisation algorithm can be formulated minimising the error between the OP and the IP measurements along the intersecting edge for each plane. The inputs needed for such a reconstruction is the inlet plane velocity field at a given time instance at which the volumetric measurement is required and sufficiently long time-sequence of snapshots of the OP. These, upon reconstruction, would then provide us with the much needed volumetric measurements from easily obtainable planar measurements. The minimisation algorithm for identifying the optimal snapshot time instance is formulated in the next section.

\subsection{Mathematical formulation}

Let the velocity field in 3D be defined as $\boldsymbol{U}(x, y, z, t)$, where in $\boldsymbol{U}$ is a vector incorporating the three components of velocity at each point in space defined by $(x, y, z)$ and $t$ defines the instant in time of the flow field. The velocity field in the OP can be expressed in this format as $\boldsymbol{U}\left(x, y, z_{0}, t\right)$, where $z_{0}$ stands for the point of intersection between the OP and the IP. Similarly, the velocity field in the IP can be expressed as $\boldsymbol{U}\left(x_{0}, y, z, t_{0}\right)$, where $x_{0}$ stands for the first point in the $\mathrm{OP}$ and $t_{0}$ stands for the time instance at which the volumetric measurement is required. For ease of understanding, the velocity on the OP common with the IP (i.e. at $x_{0}, y, z_{0}$ ) is expressed as $U_{O P}(y, t)$ and the velocity on the IP at time of reconstruction is referred to as $U_{I P}(y, z)$. In order to find the optimal instance of match between the OP observations in the plane $z=z_{0}$ and the IP observation in the plane $x=x_{0}$, a simple least square based cost function can be formed for each vertical line (along $y$ ) in the IP as a function of the spanwise direction $z$ :

$$
J(z)=\sum_{y}\left(\boldsymbol{U}_{O P}\left(y, t_{\mathrm{z}}\right)-\boldsymbol{U}_{I P}(y, z)\right)^{2}
$$


where $J(z)$ is the cost function for a given $z$, and $t_{\mathrm{z}}$, which is the parameter to optimise, is the time of observation, as a function of $z$, that matches best with the IP measurements for a given $z$. The optimisation function here accumulates the error of all three velocity components. In practice, individual optimisation procedures may be carried out for each velocity component and the results can be collated to provide the volumetric field.

The gradient of the cost function $J(z)$ along the variable of interest $t_{\mathrm{z}}$ is then given as:

$$
\partial_{t_{\mathrm{z}}} J(z)=\sum_{\mathrm{y}} 2\left[\boldsymbol{U}_{O P}\left(y, t_{\mathrm{z}}\right)-\boldsymbol{U}_{I P}(y, z)\right] \partial_{t_{\mathrm{z}}} \boldsymbol{U}_{O P}\left(y, t_{\mathrm{z}}\right),
$$

where the velocity derivative is calculated using a first order forward finite difference scheme.

A simple time stepping gradient descent method can then be envisaged as:

$$
t_{\mathrm{z}}^{n+1}=t_{\mathrm{z}}^{n}-\epsilon \partial_{t_{\mathrm{z}}} J(z)
$$

where $\epsilon$ is a suitable weight.

In order to perform a gradient descent (GD) optimisation, an appropriate starting guess $t_{\mathrm{st}}$ for $t_{\mathrm{z}}$ needs to be provided. While a random $t_{\mathrm{st}}$ can be provided, better methods can be envisaged. Two methods were implemented to identify a good $t_{\mathrm{st}}$. The first method identifies a fixed $t$ such that the summation of the total square error between OP at time $t$ and IP for all $z$ was minimal:

$$
t_{\mathrm{st}}=\min \left(\sum_{\mathrm{z}}\left(\sum_{\mathrm{y}}\left(\boldsymbol{U}_{O P}(y, t)-\boldsymbol{U}_{I P}(y, z)\right)^{2}\right)\right)
$$

Such a method could help in reducing computational cost by providing a starting guess closer to the optimal solution - for an observation dataset ranging over a long period of time, such a method could reduce computational cost considerably.

The above defined method provides a good estimate as a starting guess however, for a large spatial domain, the cost of computing this would be of the order of $n_{\mathrm{y}} \times n_{\mathrm{z}} \times n_{\mathrm{t}}$, where $n_{i}$ is the number of points along the $i$ spatial or temporal direction. The second method, which is computationally less expensive, involves an averaging along the spanwise $z$ direction of the IP $\left(\overline{\boldsymbol{U}}_{I P}(y)\right)$ and minimising the error of the OP measurements with this averaged lateral profile of the IP:

$$
\left.t_{\mathrm{st}}=\min \left(\sum_{\mathrm{y}}\left(\boldsymbol{U}_{O P}(y, t)-\overline{\boldsymbol{U}}_{I P}(y)\right)^{2}\right)\right) .
$$

The cost for such a computation is only of the order of $\left(n_{\mathrm{y}} \times n_{\mathrm{z}}+n_{\mathrm{y}} \times n_{\mathrm{t}}\right)$.

It is important to note that the use of a GD methodology could result in a fractional $t(z)$ for which observations are not available. For such cases, an interpolation of the velocity profile is performed to obtain the field at the fractional $t_{\mathrm{z}}$ - linear interpolation has been used in this manuscript when required.

\subsection{Reduced order formulation}

A full scale 3D reconstruction of a flow field can be computationally expensive while inducing strong discontinuities and introducing divergence within the flow field. A Proper Orthogonal Decomposition (POD) based reconstruction would be computationally inexpensive while providing a smoothed flow field. Here, a POD method of reconstruction is constructed similar to the full scale reconstruction explained in the previous section. Two different POD can be envisaged on the given flow field. One is along the $1 \mathrm{D}$ vertical $(y)$ direction for identifying the 
best match - this would reduce the computational cost of optimisation. The second is a 2D POD of the OP velocity facilitating a large scale reconstruction resulting in a smoothed flow field. The two POD formulations are described further in the following sections.

\subsubsection{D-3C POD - matching}

Let the flow field along $x_{0}$ in the OP be decomposed as:

$$
\boldsymbol{U}_{O P}^{\prime}(y, t)=\sum_{i=1}^{N} \boldsymbol{\phi}_{\mathrm{i}}^{1 D}(y) \mathrm{a}_{\mathrm{i}}^{1 D}(t)
$$

where $\boldsymbol{U}_{O P}^{\prime}(y, t)=\boldsymbol{U}_{O P}(y, t)-\overline{\boldsymbol{U}}_{O P}(y)$, with $\overline{\boldsymbol{U}}_{O P}(y)$ being the time averaged quantity, $N$ is the total number of modes, $\phi_{\mathrm{i}}^{1 D}$ is the POD spatial modes, and $\mathrm{a}_{\mathrm{i}}^{1 D}$ are the time coefficients for a given $t$.

Considering the turbulence homogeneity assumption along the spanwise direction, the IP flow field can also be expressed on the same spatial modes obtained from the OP decomposition.

$$
\boldsymbol{U}_{I P}^{\prime}(y, z)=\sum_{i=1}^{n} \phi_{\mathrm{i}}^{1 D}(y) \mathrm{a}_{\mathrm{i}}^{\prime D}(z),
$$

where $\mathrm{a}_{\mathrm{i}}^{1 D}(z)$ represents the time coefficients for the flow field along the lateral direction for each spanwise $z$ coordinate and thus is expressed as a function of $z$ rather than time $t$. With the two sets of time coefficients, based on the same set of spatial modes, a GD minimisation algorithm can be formulated, similar to the full scale reconstruction, comparing the time coefficients instead of the flow field:

$$
J(z)=\sum_{i=1}^{n}\left(\mathrm{a}_{\mathrm{i}}^{1 D}\left(t_{\mathrm{z}}\right)-\mathrm{a}_{\mathrm{i}}^{\prime 1 D}(z)\right)^{2},
$$

where $J(z)$ is the cost function, and $n$ stands for the subset of modes considered from the $N$ modes global set. A gradient of this cost function is then given as:

$$
\partial_{t_{\mathrm{z}}} J(z)=\sum_{i=1}^{n} 2\left[\mathrm{a}_{\mathrm{i}}^{1 D}\left(t_{\mathrm{z}}\right)-\mathrm{a}_{\mathrm{i}}^{\prime 1 D}(z)\right] \partial_{t_{\mathrm{z}}} \mathrm{a}_{\mathrm{i}}^{1 D}\left(t_{\mathrm{z}}\right) .
$$

Similar methods can be used for the starting guess $t_{\mathrm{z}}$ as enumerated in the full scale reconstruction. Such a decomposition will provide a decrease in computation cost of the minimisation algorithm, however, it would still lead to a full scale reconstruction of the flow field. This reconstruction could have strong discontinuities as well as non-negligible divergence. To attenuate this, a large-scale reconstruction of the flow can be done by performing a POD on the 2D OP measurements and using only the high-energy modes.

\subsubsection{D-3C Planar POD - reconstruction}

With the optimal time $t_{\mathrm{z}}^{\text {opt }}$ obtained from the 1D POD matching algorithm or from the full scale algorithm, the reconstruction of the $3 \mathrm{D}$ domain can be done using $2 \mathrm{D}$ POD. Let the $2 \mathrm{D}$ OP flow field be decomposed as:

$$
\boldsymbol{U}_{O P}^{\prime}(x, y, t)=\sum_{i=1}^{N} \phi_{\mathrm{i}}^{2 D}(x, y) \mathrm{a}_{\mathrm{i}}^{2 D}(t),
$$


where $\boldsymbol{U}_{O P}^{\prime}(x, y, t)=\boldsymbol{U}_{O P}(x, y, t)-\overline{\boldsymbol{U}}_{O P}(x, y)$, and $\overline{\boldsymbol{U}}_{O P}(x, y)$ is the average along time.

The reconstruction of the $3 \mathrm{D}$ velocity $\left(U_{r e c}\right)$ can then be obtained used the time coefficients of the snapshot at the optimal time in the sequence.

$$
\boldsymbol{U}_{r e c}\left(x, y, z, t_{0}\right)=\overline{\boldsymbol{U}}_{O P}(x, y)+\sum_{i=1}^{n} \boldsymbol{\phi}_{\mathrm{i}}^{2 D}(x, y) \mathrm{a}_{\mathrm{i}}^{2 D}\left(t_{\mathrm{z}}^{\mathrm{opt}}\right) \forall z
$$

Depending on the number of modes considered $(n)$, a smooth field or a full scale representation can be obtained for $\boldsymbol{U}$.

\subsection{Averaging SO method}

For a given line on the IP, there may be multiple optimal time-instances of OP snapshots whose corresponding error varies only marginally from one another. By performing the optimisation as described above, we identify only one such optimal time-instance which is used to recreate the 3D velocity field. Such a reconstruction will realistically provide a velocity field that has minimal error but with significant noise/discontinuities due to the violation of continuity between subsequent velocity fields in the spanwise direction. This could be possibly redressed by identifying and averaging multiple time-instances depending on a user-defined error threshold as a percentage of the minimal error obtained from the optimisation procedure. For such a methodology (termed as 'Averaging SO method'), the reconstructed velocity field can be expressed as,

$$
\boldsymbol{U}_{r e c}\left(x, y, z, t_{0}\right)=\frac{\sum_{i=1}^{n_{z}} \boldsymbol{U}_{O P}\left(x, y, t_{z}^{i}\right)}{n_{z}} \forall z,
$$

where $n_{z}$ is the number of optimal snapshots identified by the algorithm for each $z$ within the user-defined tolerance, and $t_{z}^{i}$ is the time of each optimal snapshot from $1-n_{z}$ for each $z$.

\section{Application and Results}

The full-scale reconstruction algorithm is applied first to two synthetic cases at moderate Re: (i) Wake flow around a cylinder at Re 3900 (section 3.1.1), and (ii) channel flow at friction velocity based Re of 590 (section 3.1.2). This is followed by an application to three experimental data sets: (i) Wake flow around a cylinder at Re 300 (section 3.3.1), (ii) Wake flow around a cylinder at Re 3900 (section 3.3.2), and (iii) mixing layer with $\lambda$ of 0.33 (section 3.3.3). This section concludes with an analysis of variations of the SO algorithm and post-processing techniques on the reconstructed volumetric data. Details of the five cases are provided in table 2.

\subsection{Synthetic Case-studies}

\subsubsection{Wake flow around a circular cylinder at Re 3900}

For the synthetic case of wake flow around a circular cylinder at Re 3900, the input data are obtained from an LES simulation of the flow using the flow solver Incompact3d (Laizet \& Li, 2011; Chandramouli et al. 2018). A domain measuring $20 D \times 20 D \times \pi D$, where $\bar{D}$ stands for the diameter of the cylinder, is simulated with $241 \times 241 \times 48$ mesh points with a time-step of 0.003 non-dimensional time. The mesh along the $y$-direction is stretched with more points near the centre of the domain. A total of $25003 \mathrm{D}$ velocity snapshots are stored every 50 time-steps corresponding to $\sim 75$ vortex sheddings after an initialisation period of 15 vortex sheddings with no data-collection.

The 3D snapshots are pre-processed to extract the $2 \mathrm{D}$ information on the IP and the OP shown in figure 1. The IP, measuring $L_{\mathrm{y}} \times L_{\mathrm{z}}=4 D \times \pi D$, is located $1.66 D$ behind the trailing edge of 
Table 1: Summary of synthetic and real configurations.

\begin{tabular}{lccccccc}
\hline Case & $R e$ & Snapshots & $\Delta t U / L^{1}$ & $\left(L_{y} \times L_{z}\right) / L$ & $\left(L_{x} \times L_{y}\right) / L$ & $n_{y} \times n_{z}$ & $n_{x} \times n_{y}$ \\
\hline 1 - LES wake & 3900 & 2500 & 0.003 & $4 \times \pi$ & $5 \times 4$ & $130 \times 48$ & $60 \times 130$ \\
2 - DNS channel & 590 & 1000 & 0.00125 & $2 \times \pi$ & $2 \pi \times 2$ & $257 \times 384$ & $384 \times 257$ \\
3 - PIV wake 300 & 300 & 4000 & 0.12 & $4.3 \times 6.4$ & $8.4 \times 4.3$ & $72 \times 108$ & $145 \times 72$ \\
4 - PIV wake 3900 & 3900 & 20540 & 0.258 & $7 \times 5.6$ & $4.3 \times 7$ & $93 \times 72$ & $64 \times 93$ \\
5 - PIV mixing layer & 1540 & 4000 & 0.015 & $2.4 \times 2.7$ & $2.4 \times 2.4$ & $100 \times 115$ & $100 \times 100$ \\
\hline
\end{tabular}

\footnotetext{
${ }^{1} L$ stands for the cylinder diameter $D$, for the channel height $H$ or for the mixing layer vorticity thickness $\delta_{\omega}$.
}

the cylinder. The OP, measuring $L_{\mathrm{x}} \times L_{\mathrm{y}}=5 D \times 4 D$, intersects the IP at the mid-point of the spanwise direction $(z=1.57 D)$. The IP is obtained corresponding to the first instance in the OP time-sequence. A sample streamwise velocity field on the IP and OP is shown in figure 3 .
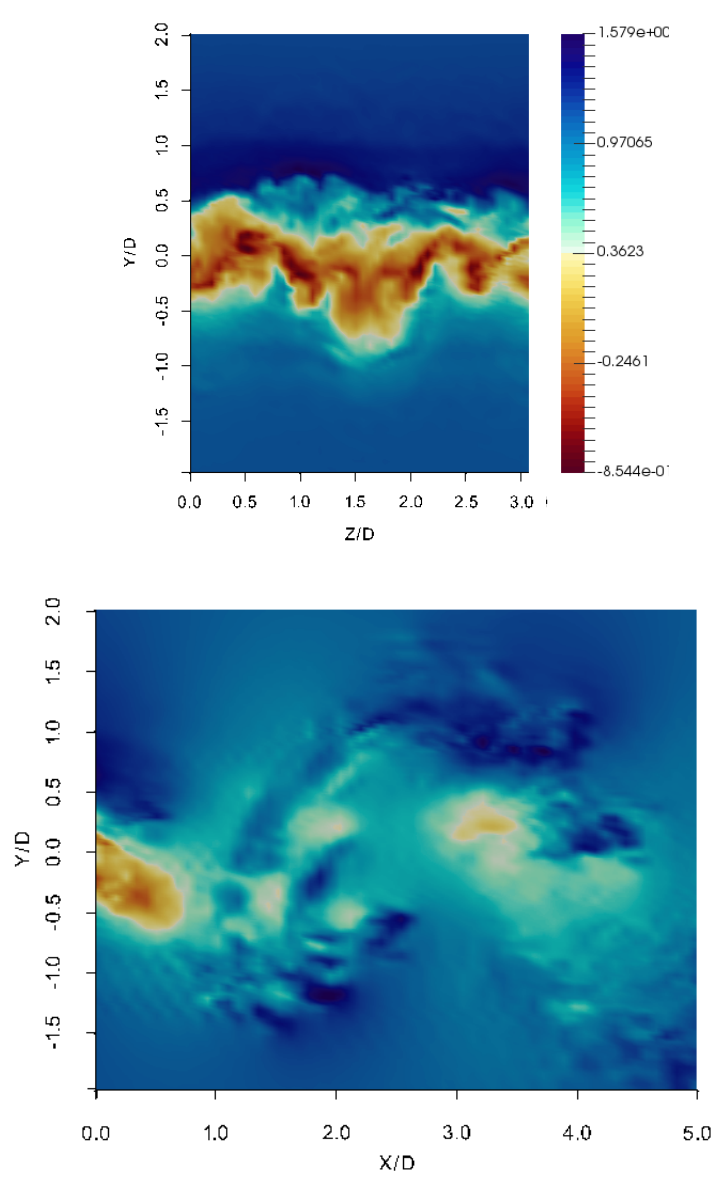

Figure 3: Instantaneous streamwise velocity fields extracted on the IP and the OP from the LES of wake flow.

The IP reconstruction from the algorithm is shown in figure 4. The contour maps of a given velocity component for a given flow are on the same colour bar and thus, the colour bar has been omitted from the comparative figures (see figure 4, for example) for compactness. The biggest advantage of such a reconstruction algorithm is the time taken for the reconstruction 


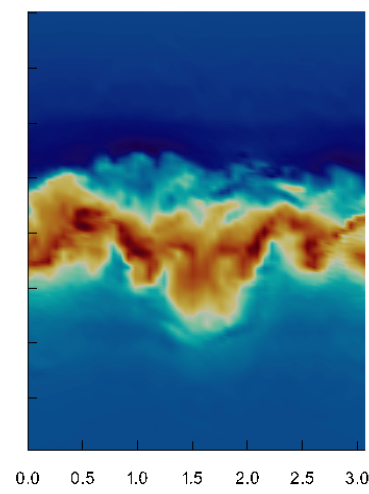

Z/D

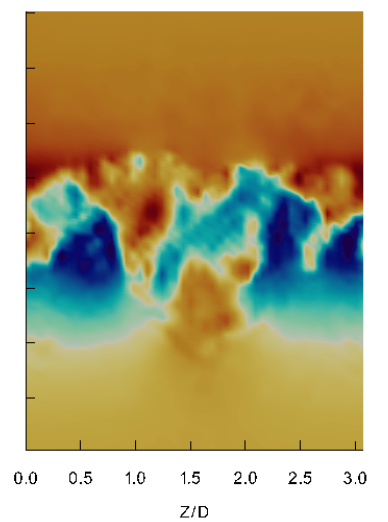

$\mathrm{Z} / \mathrm{D}$

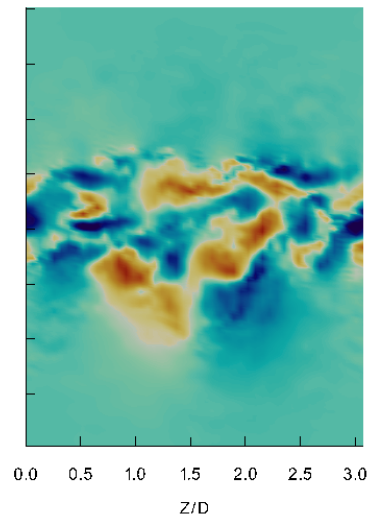

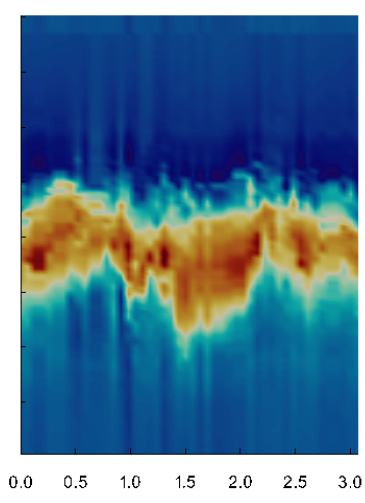

ZID

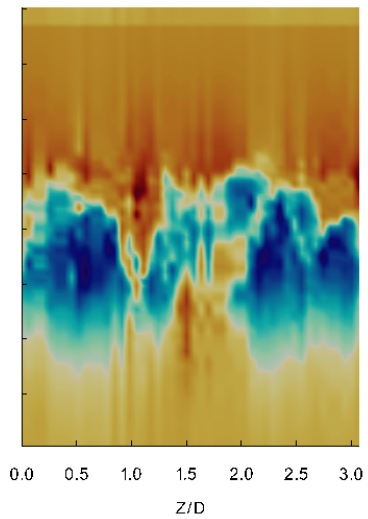

$\mathrm{ZID}$

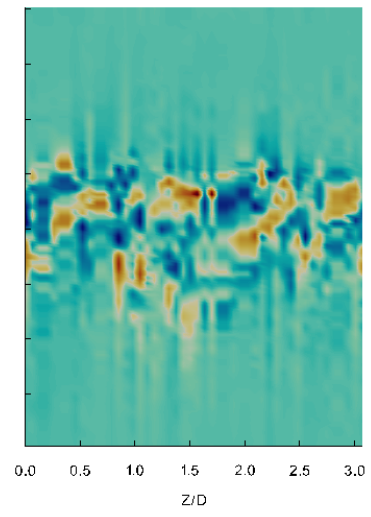

Figure 4: LES wake flow instantaneous velocity fields extracted on the IP. From top to bottom: streamwise, vertical and spanwise velocity components. From the left to the right: reference and reconstruction.

$\left(t_{\mathrm{rec}}\right)$ which for this case was $t_{\mathrm{rec}}=14.96 \mathrm{~s}$. This time is independent of the number of OP snapshots considered or the type of flow. It is only dependant on the discretisation of the intersection line between the OP and the IP. A finer discretisation of this line would increase the computational cost of the algorithm and vice-versa. The ability of the algorithm to capture the main structures of the flow is seen in figure 4. Just from a single plane observation timesequence, the IP has been reconstructed capturing all large scale structures of the flow in all three velocity directions. The small scale structures are also captured, however, this is attenuated by a noise and discontinuities in the reconstruction. Such a reconstruction provides a much better estimate of the 3D flow domain as compared to simplistic techniques such as mirror-imaging or interpolation.

The availability of 3D reference velocity field allows us to study the 3D reconstruction efficiency of the algorithm by comparing the velocity iso-contours (see figure 5). The reconstructed 3D 

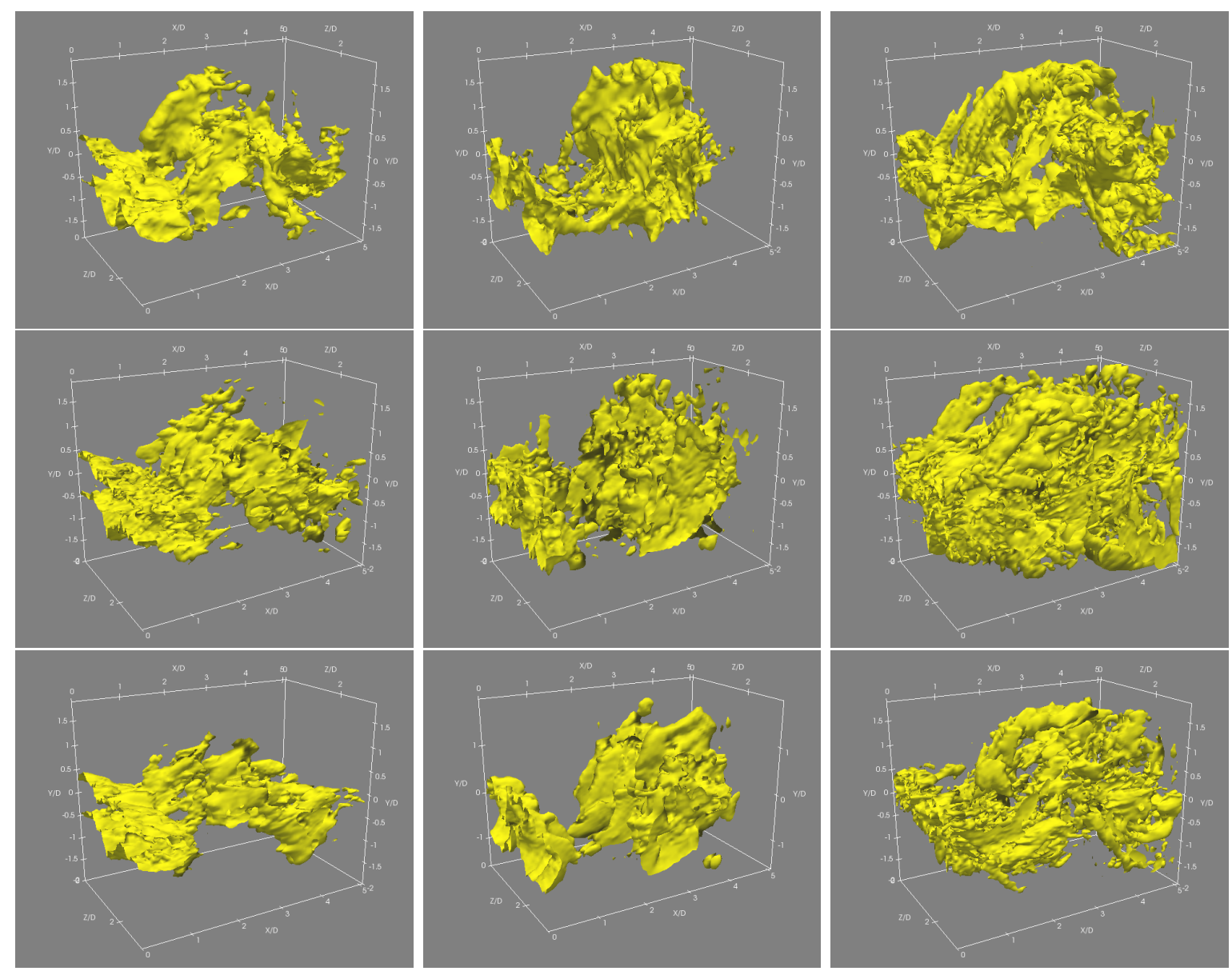

Figure 5: LES wake flow instantaneous velocity iso-contours extracted on the 3D domain. From top to bottom: reference, SO reconstruction and averaging SO reconstruction. From the left to the right: streamwise, vertical and spanwise velocity components.

velocity accurately captures the large-scale motions of the flow. The location of the vortex street is captured well in the reconstruction iso-contours. There is significant introduction of noise especially in the spanwise velocity component but the large scale-correlation of vortex structures is efficiently represented. Considering the computational efficiency and simplicity of the algorithm, the results obtained advocate towards the efficacy and applicability of the algorithm as a preliminary reconstruction tool to obtain 3D motion fields from cross-plane observations. The case study corresponding to the averaging $\mathrm{SO}$ reconstruction method is explained in $\$ 3.2 .2$. The iso-contours are presented here for ease of comparison and to avoid repetition.

\subsubsection{Channel flow at $R e_{\tau} 590$}

A direct numerical simulation (DNS) is performed for channel flow at $\operatorname{Re}_{\tau} 590$ using Incompact3d from which the observations are extracted for the reconstruction algorithm. The DNS is performed on a domain measuring $12.56 H \times 2 H \times \pi H$ discretised into $768 \times 257 \times 384$ meshpoints with a time-step of 0.00125 non-dimensional time. Considering the computational cost and memory requirements of the DNS, only a limited set of 1000 snapshots could be obtained spaced 50 time-steps apart. The collection of the snapshots begins after the flow is converged. This case-study is a good example to analyse the performance of the algorithm under limited observation snapshots. However, considering the availability of $3 \mathrm{D}$ data-set, multiple OP can 
be defined and velocity fields from parallel OPs can be extracted. This alleviates the issue of insufficient snapshots and provides an opportunity to study the performance of the algorithm with respect to the size of the OP snapshots. Three sub-cases are studied with OP data extracted from $1(1 \mathrm{p}), 5(5 \mathrm{p})$, and $10(10 \mathrm{p})$ parallel planes - 10 planes consist of only $2.5 \%$ of the total spanwise set of planes.

The IP is placed in the centre of the channel measuring $L_{\mathrm{y}} \times L_{\mathrm{z}}=2 H \times \pi H$ discretised with the same number of points as the DNS, i.e. $257 \times 384$. The OP begins at the IP and extends to the end of the channel with the intersection at $z=[1.57 H]$ for $1 \mathrm{p}, z=$ $[0.52 H, 1.04 H, 1.57 H, 2.09 H, 2.62 H]$ for $5 \mathrm{p}$, and for $10 \mathrm{p} z=[0.26 H, 0.52 H, 0.78 H, 1.04 H, 1.3 H, 1.57 H, 1.83 H$, $2.09 H, 2.35 H, 2.62 H]$. The OP is discretised into $N_{\mathrm{x}} \times N_{\mathrm{y}}=384 \times 257$ mesh-points. The reference inlet streamwise velocity and a sample OP measurement for the streamwise velocity is shown in figure 6 .
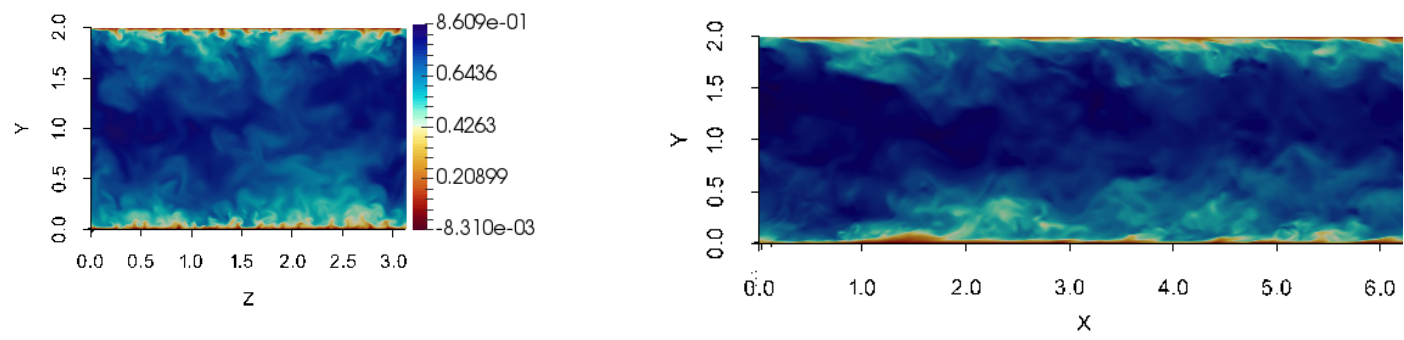

Figure 6: Instantaneous streamwise velocity fields extracted on the IP and the OP from the DNS of channel flow.

The reconstructed velocity fields on the IP are shown in figure 7. The reconstruction time for $1 \mathrm{p}$ case, as shown in table 2, is $t_{\mathrm{rec}}=257.12 \mathrm{~s}$ - the higher time is due to the finer resolution of the DNS. From the reconstructed images, it is clear that small scale structures of the DNS are not entirely reconstructed by the algorithm for the vertical $(v)$ and spanwise $(w)$ velocity profiles. However, for all velocity components, near the wall where important turbulent phenomena occur, the algorithm reconstructs with good accuracy the flow features. A significant improvement in the reconstructed velocity fields can be seen with $5 \mathrm{p}$ reconstruction as compared to the $1 \mathrm{p}$. A further increase to $10 \mathrm{p}$ does not provide a drastic improvement but better representation of the small scale structures can be observed, mainly near the wall. This suggests that a minimum set of $\mathrm{OP}$ snapshots are required in order to obtain a good reconstruction and any further increase tends to improve the output further albeit at a reduced rate.

\subsection{Algorithm Enhancement Techniques}

This section explores the capabilities of the POD and averaging variants of the SO technique towards obtaining a smooth reconstruction. In addition, the ability of the algorithm to deal with noisy synthetic observations is also analysed while a simulation-based technique is analysed for removing divergence from the reconstructed volume. All these techniques are applied to the case study of synthetic wake flow at Re 3900 and the results are presented on this flow.

\subsubsection{POD based SO method}

The mathematical formulation for the POD based SO method, provided in section 2.3, is applied here to wake flow around a circular cylinder at Re 3900 (case-study 1 in table 2). Both a 1D POD based matching algorithm and a 2D POD based reconstruction is performed and the reconstructed volume is presented in figure 8. The first 72 modes are used for the POD 

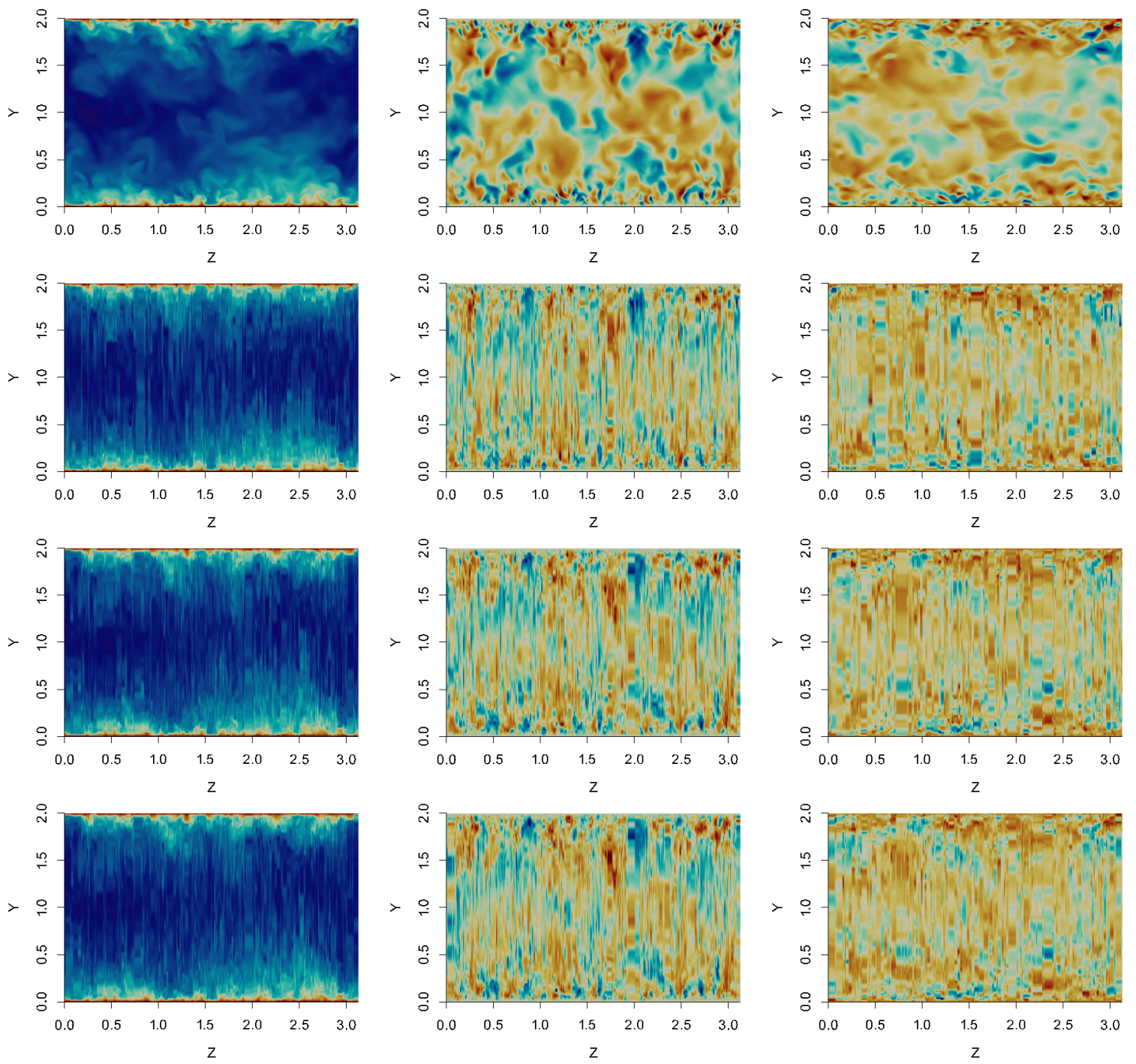

Figure 7: DNS channel flow instantaneous velocity fields extracted on the IP. From top to bottom: reference, reconstruction with 1 plane, reconstruction with 5 planes, and reconstruction with 10 planes. From the left to the right: streamwise, vertical and spanwise velocity components. 

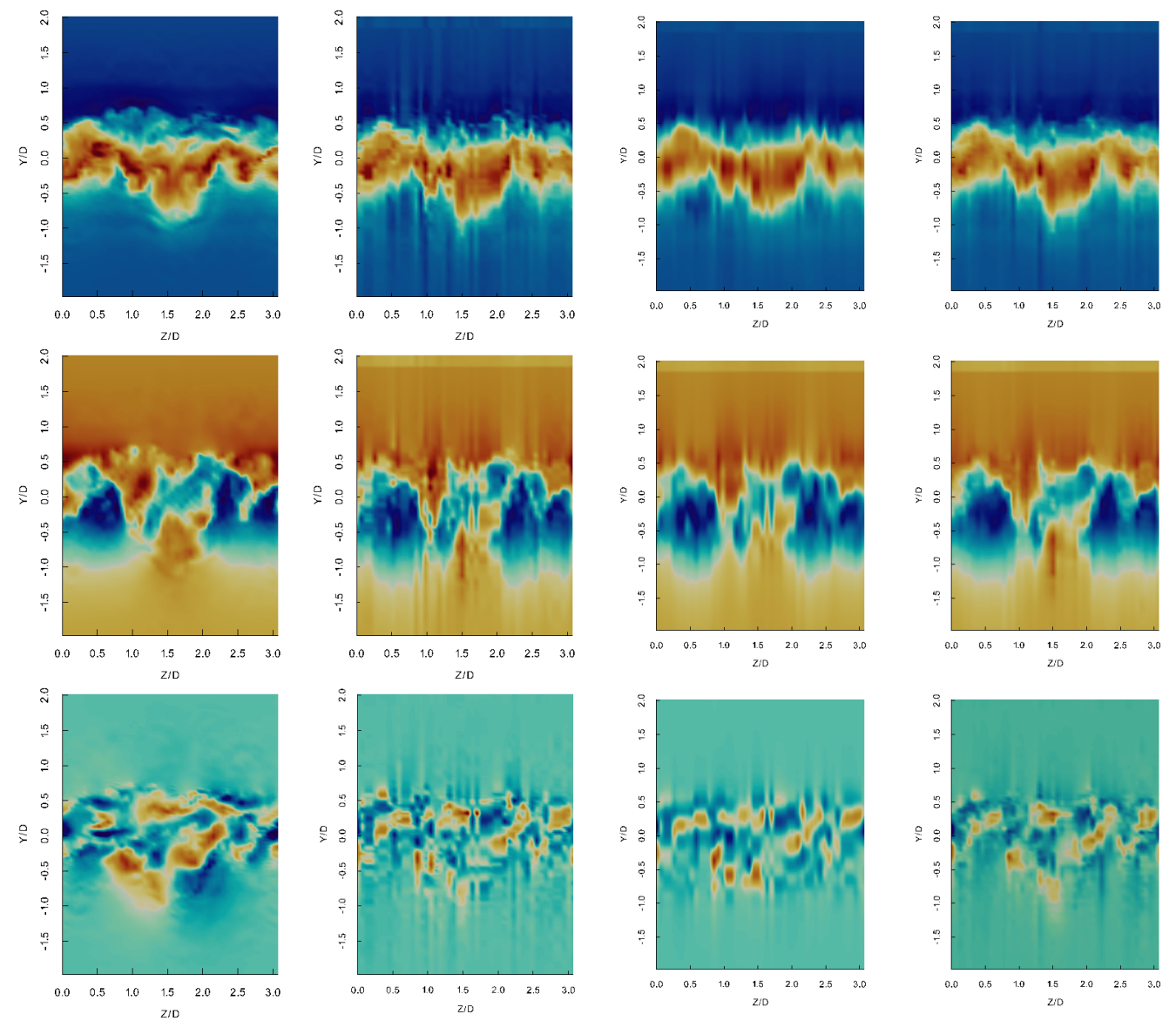

Figure 8: Reconstructed velocity fields using POD based SO method and averaging SO method. From top to bottom: streamwise, vertical, and spanwise velocity component. From left to right: reference, SO reconstruction, POD based SO reconstruction, averaging SO reconstruction.

matching while only the first 20 modes are used in the reconstruction phase - the reconstruction process is faster taking only $t_{\text {rec }}=1.6 s$ for the reconstruction. The POD based reconstructed provides a smooth representation of the velocity fields thus reducing discontinuities seen in the full reconstruction. However, this is associated to a reduced recovery of the small-scale structures in the POD reconstruction. Such a reconstructed field could be used as a reference, so called background initial condition, in variational data-assimilation procedures as it provides a smooth $3 \mathrm{D}$ velocity field based on the observations from which the optimisation procedure for the DA can begin.

\subsubsection{Averaging SO method}

A closer look at POD reconstruction results (in figure 8) suggests that there maybe not be just one optimal time for a given $\mathrm{z}$ plane. The time instances obtained from the full-scale reconstruction and the POD based reconstructed vary slightly for certain z planes. The availability of multiple optimal time-instances led to the concept of averaging SO method. In this method, for each spanwise plane, all time-instances below a certain error threshold are averaged to provide the final velocity reconstruction. The utility of this method lies in reduction of spurious oscillations and removal of spanwise discontinuities due to averaging procedures similar to the POD. The error threshold for each plane is set as optimal error for the corresponding plus a $10 \%$ bias. The results of the averaging SO method is shown in figure 8 . The averaging method performs similar to the POD based SO method, however, a higher recovery of the small scale 
structures are obtained with the averaging method compared to POD as well as a significant improvement in spanwise continuity. The reconstruction of the averaging SO method is better than the full-scale reconstruction with improved recovery of turbulent structures and minimal spatial discontinuities.

Both the averaging SO method and the POD based SO method help reduce discontinuities within the reconstruction. The POD method is an ideal alternative for fast computations $(\sim$ 1.6 seconds) as the optimisation is done on a smaller set of modes as compared to the full-scale reconstruction. The averaging SO method, however, appears to give better results compared to the POD at the cost of higher computational time ( $~ 8.1$ seconds) as well as needing, ideally, more number of OP snapshots to find multiple optimal snapshots. While for this specific case the additional time taken may not be high, for larger dimensional systems searching over a larger time-sequence of data, the difference between the two method may be high.

A 3D comparison is performed with the velocity iso-contours of the averaging SO method, and the simple SO method from section 3.1.1 with the reference field in figure 5. The reduction of noise can be seen with the use of the averaging procedure as compared to the simple SO algorithm. In addition, a better correlation between structures is also seen with the averaging method. However, coherent small-scale structures are lost with the application of the averaging method that are seen in the reference - this is clearly seen in the streamwise velocity iso-contours where the rising vortex structure of the reference is seen in the simple reconstruction but not the averaged.

\subsubsection{Noisy data-sets}

The algorithm is capable of reconstructing the volumetric domain provided perfect synthetic data-sets. However, the main application of the algorithm is towards experimental data-sets which, in general, tend to be noisy. Before applying the algorithm to PIV data-sets in section 3.3 with unquantified noise, we test the algorithm with the synthetic data-set with artificially added noise. A $10 \%$ white noise, parametrised by $\boldsymbol{U}_{\max }$ for each component, is added to the IP and OP snapshots while maintaining all other parameters for the flow. With this noisy dataset, the reconstruction is performed using both the SO and the averaging SO methodology on the volume. The corresponding reference, and reconstructed velocity fields are shown in figure 9

The modulation of the reference field due to the noise can be clearly seen in figure 9. The amount of noise added (10\%) is comparatively higher than the noise level as obtained from modern experimental techniques such as PIV. Despite the noisy IP and OP, both variants of the algorithm are capable of reconstructing the major physical structures in the flow. With the SO algorithm, the noise is propagated onto the reconstructed volume (as can be visibly seen in the streamwise component). However, a significant reduction is noise is observed when the averaging $\mathrm{SO}$ algorithm is used to reconstruct the volume. The average performed over multiple snapshots tends to reduce the noise-level providing a smooth representation of the flow field.

\subsubsection{Divergence-free reconstruction}

The flows considered in this work are incompressible fluid flows and thus are divergence free. However, the process of reconstruction provides a final volumetric data-set which is clearly not divergence free. In this sub-section a few possible methods for producing divergence free data-sets by post-processing are discussed. 

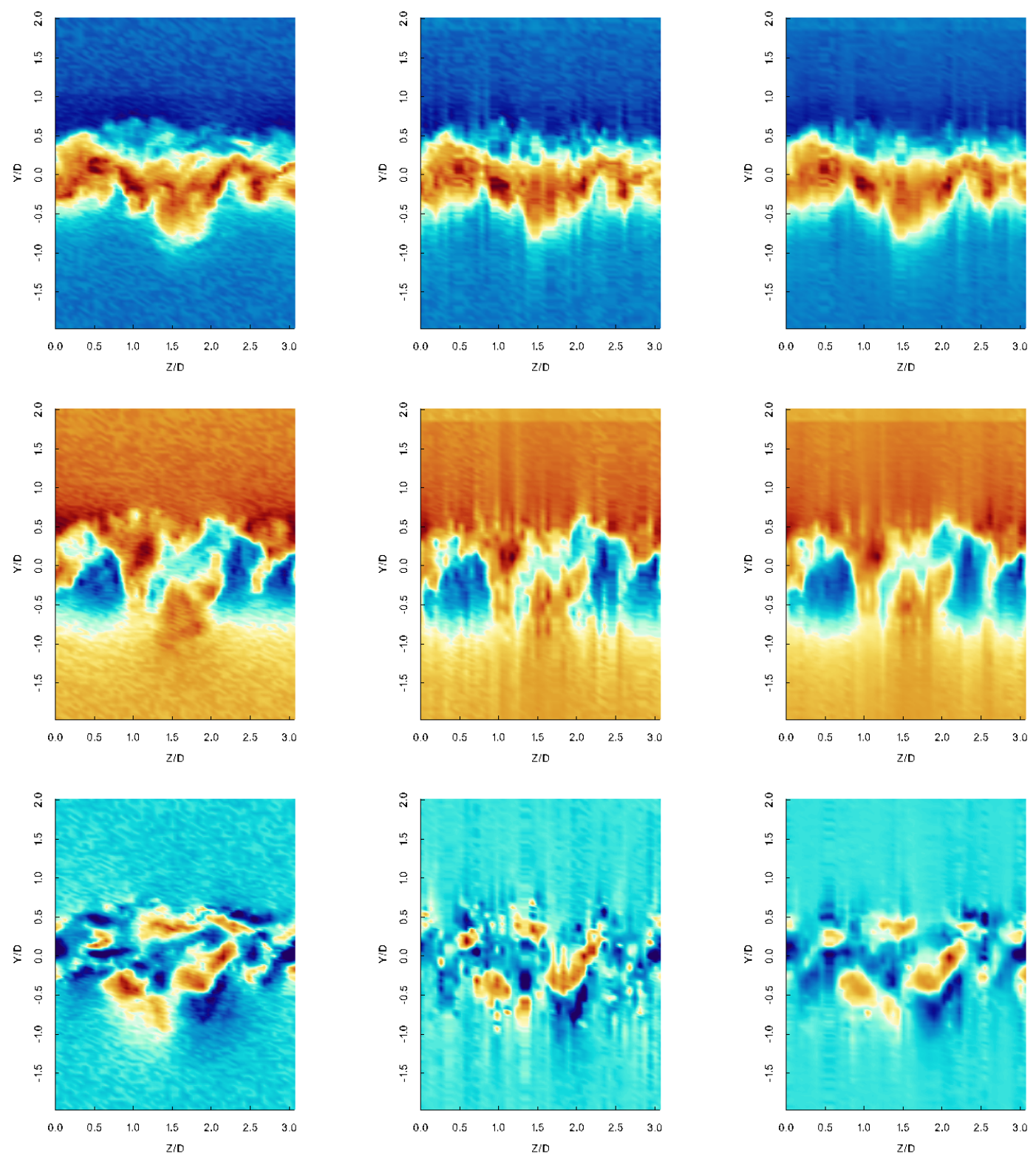

Figure 9: Reconstructed velocity fields using SO method and averaging SO method with noisy synthetic data-sets for wake flow around a cylinder at 3900. From top to bottom: streamwise, vertical, and spanwise velocity component. From left to right: reference with added noise, SO reconstruction, averaging SO reconstruction. 
An incompressible fluid flow solver, such as incompact3d, maintains a divergence free flow by the action of the pressure-poisson equation where the divergence present in the flow is used to modify the pressure which successively modifies the velocity field to be divergence free using a fractional step method (Laizet \& Lamballais, 2009). Thus, performing a simulation, using such a flow solver with the reconstructed velocity field as the initial condition, for a single time-step would provide a divergence free velocity field with an associated pressure containing the divergence.

The reconstruction for the synthetic wake flow at Re 3900 has been simulated with incompact3d to produce such a divergence-free field - the final divergence of the flow was of the order of $10^{-14}$ i.e. numerical accuracy. The divergence field before and after simulation with Incompact3d is shown in figure 10. The stability of the reconstruction was also analysed by simulating further the flow for a limited number of time-steps and the flow was indeed stable when initialised with the reconstructed velocity fields. This is necessary in order to use the results of the SO method in variational DA as the background state.

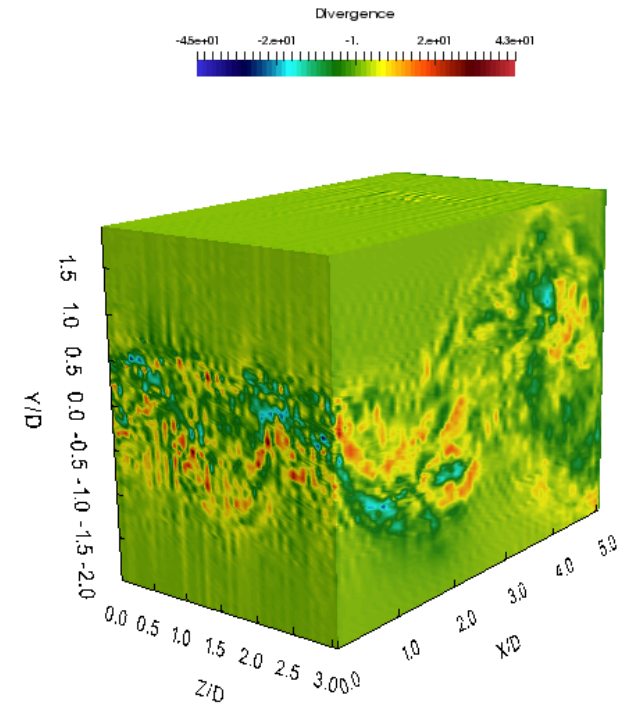

Before

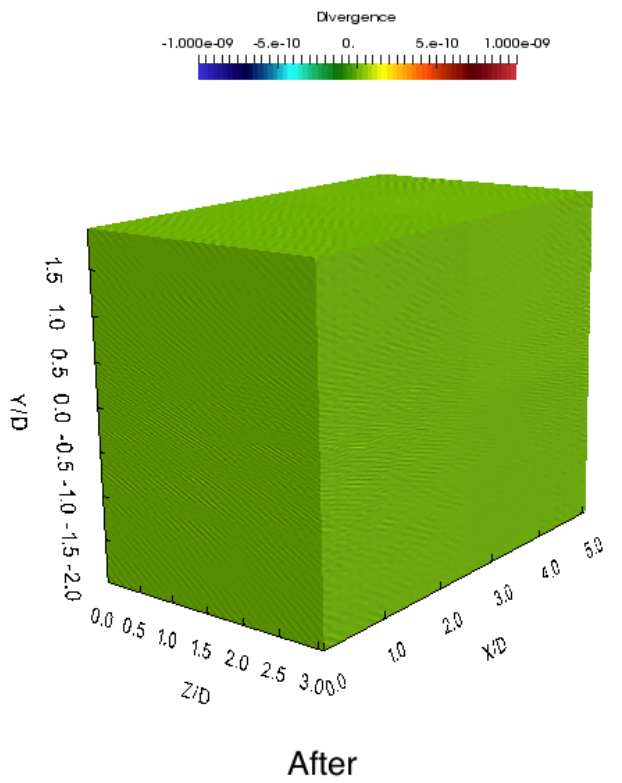

After

Figure 10: Divergence of the flow field before and after simulation with Incompact3d.

An alternative to produce a divergence free reconstruction is to use the Helmholtz decomposition (Van Bladel, 1958). Helmholtz theorem states that "A continuous vector field, a, can be decomposed into the sum of a gradient and a curl term", i.e. a velocity field can be split as:

$$
\bar{U}=-\nabla \phi+\nabla \times \psi
$$

where $\bar{u}$ is the velocity field, $\phi$ is the scalar potential and $\psi$ is the vector potential. The solenoidal $U_{s o l}$ and irrotational $U_{i r r}$ part of velocity can hence be expressed as:

$$
U_{\text {sol }}=\nabla \times \psi ; \quad U_{i r r}=-\nabla \phi
$$

Using simple algebraic manipulations, the scalar potential can be calculated following which the irrotational field, which contains the divergence, can be calculated and removed from the true field. Such a manipulation can be easily done in the Fourier space. The resultant vector field would contain minimal divergence as compared to the original field. However, as a part of the original field was removed, the energy of the resultant field would be lower than the original and this difference needs to be compensated for. 
An alternative methodology towards reconstructing a divergence-free flow is to use the divergence correction scheme (DCS) as developed by de Silva et al. (2013). This method uses a constraint-based non-linear optimisation technique to reduce the divergence of a given velocity field to near zero. An improvement to this technique was provided by Wang et al. (2016) called the divergence-free smoothing (DFS) method which reduces divergence while also smoothing the velocity field to remove outliers and missing vectors. A more comprehensive approach would be to use the modified pressure correction scheme (PIV-PCS) of Wang et al. (2018) where in the PIV data are combined with the incompressible Navier-Stokes equations to improve the data-set. The DCS and DFS family of methods provide an interesting approach towards obtaining divergence free flow fields. A combination of the SO methodology with such divergence-reducing schemes provides an avenue of interesting future work.

It is to be noted that the main application of this algorithm is towards experimental stereoscopic PIV data-sets. These data-sets tend to be a large-scale representation of the flow due to the use of interrogation windows i.e. similar to the LES case-study presented and the algorithm could be expected to reconstruct accurately the large-scale volumetric data from such data-sets. The performance of the algorithm with such experimental data-sets is the focus of the next section.

\subsection{Experimental Case-studies}

The experiments were conducted in two wind tunnels located at the Irstea regional center in Rennes, France. One open wind tunnel was used to simulate both a wake flows and mixing layers (case 3 and 5 respectively in table 2 ) in a test section measuring $3 \mathrm{~m} \times 1 \mathrm{~m} \times 1 \mathrm{~m}$. The other closed-loop wind tunnel was used to simulate a wake flow (case 4 in table 2 ) in a test section measuring $2.4 \mathrm{~m} \times 0.28 \mathrm{~m} \times 0.28 \mathrm{~m}$.

Two perpendicular plane 2D3C PIV measurements are taken in the test sections as shown in figure 1. The origin of the coordinate system is defined at the intersection between the cross planes, with $x, y$ and $z$ directed towards the streamwise, transverse and spanwise direction, respectively. A stereo PIV system was carried out to measure the three velocity components in both planes with a Litron laser system (Energy pulse of $15 \mathrm{~mJ}$ at $3,000 \mathrm{kHz}$ ) and two high speed cameras Phantom M310 (CMOS size of $800 \times 1280$ px). The flows were seeded by smoke generated from oil. The resulting image pairs were analysed by Davis software from LaVision. The specifics of the setup for each case-study is explained further in the corresponding sections.

\subsubsection{Wake flow around a circular cylinder at Re 300}

A cylinder of diameter $D=10 \mathrm{~mm}$ and length $300 \mathrm{~mm}$ was placed in the air flow. Two thin end-plates were used with specifications provided by Stansby (1974) to avoid the boundary layer effects of the walls of the test-sections. The end plates were separated by a distance of $300 \mathrm{~mm}$ with the clearance between the plates and the walls of the wind tunnel kept at $350 \mathrm{~mm}$. The free stream velocity was set at $U=0.48 \mathrm{~m} / \mathrm{s}$ corresponding to a Re of 300 . On both the IP and the OP, 4000 image pairs were acquired at a frequency of $500 \mathrm{~Hz}$. The IP was placed $19 D(190 \mathrm{~mm})$ downstream of the cylinder. The OP intersects the IP through the middle with a $10 \mathrm{~mm}$ protrusion behind the IP. The IP is discretised into $N_{\mathrm{y}} \times N_{\mathrm{z}}=349 \times 268$ mesh-points while the OP is discretised into $N_{\mathrm{x}} \times N_{\mathrm{y}}=280 \times 347$ mesh-points. Due to the presence of end-plates as well as the divergent nature of the OP, the data measured on the borders of the planes can be erroneous. In addition, the presence of extensive laminar regions in a snapshot reduces the effectiveness of the algorithm as it reduces the weight of the turbulent region. Thus only a sub-set of the original snapshot was considered for the optimisation procedure. The IP sub-set measures $L_{\mathrm{y}} \times L_{\mathrm{z}}=4.3 D \times 6.4 D$ containing $N_{\mathrm{y}} \times N_{\mathrm{z}}=72 \times 108$ mesh-points. The OP sub-set measures $L_{\mathrm{x}} \times L_{\mathrm{y}}=8.4 D \times 4.3 D$ discretised into $N_{\mathrm{x}} \times N_{\mathrm{y}}=145 \times 72$ points. A sample streamwise velocity field on the IP and the OP is shown in figure 11 

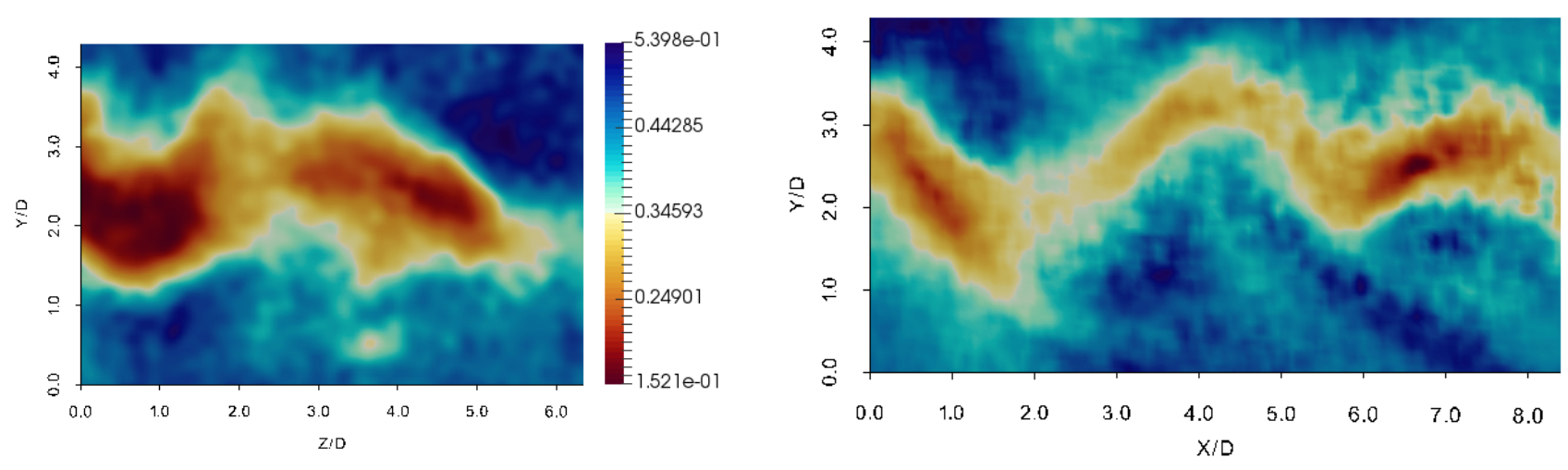

Figure 11: Instantaneous streamwise velocity fields extracted on the IP and the OP from PIV data-set of wake flow around a cylinder at Re of 300 .
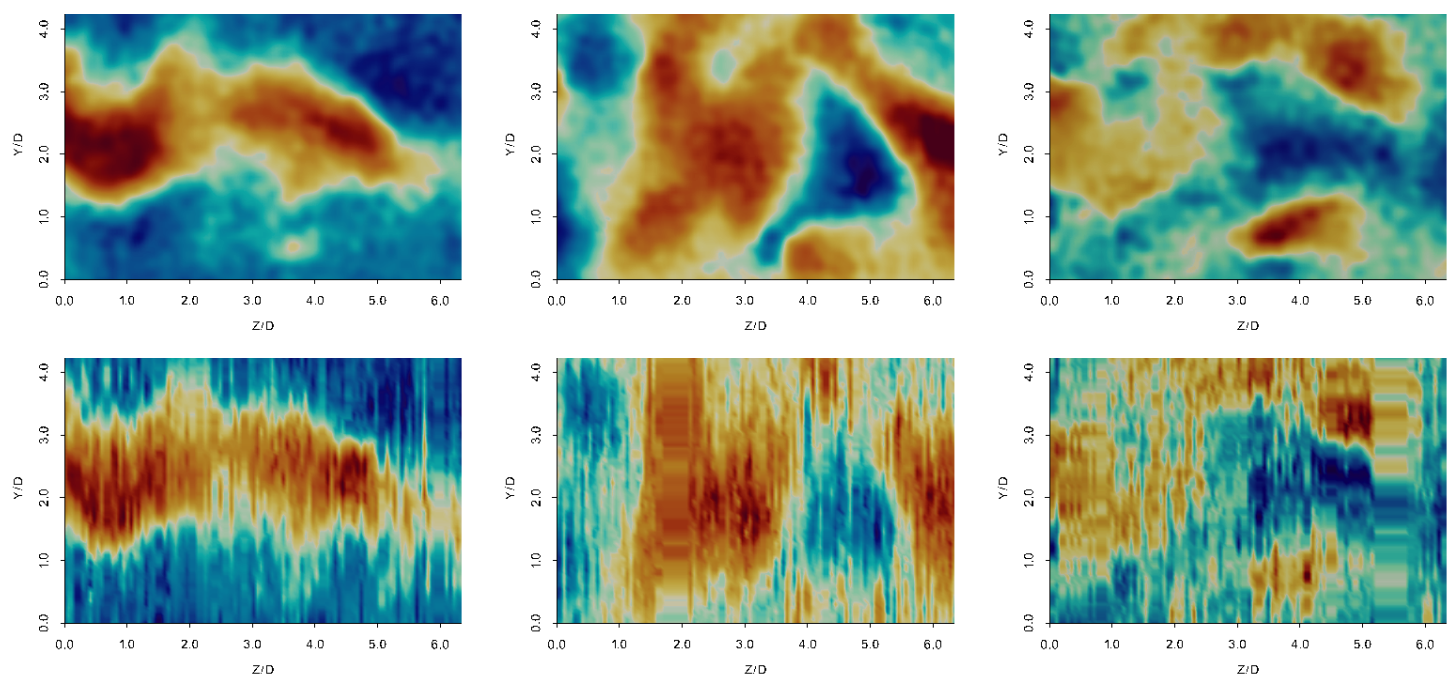

Figure 12: PIV wake flow instantaneous velocity fields extracted on the IP for Re of 300. From top to bottom: reference and reconstruction. From the left to the right: streamwise, vertical and spanwise velocity components.

The reconstructed velocity fields on the IP are shown in figure 12 along with the reference IP velocity fields. A run-time of $t_{\mathrm{rec}}=4.85 \mathrm{~s}$ was clocked by the algorithm for this case-study. Contrary to the synthetic data-sets, the experimental observations provide a noisy velocity field. However, the main structures of the flow are still captured well by the algorithm in all three principal directions. The main large scale structures in the vortex wake are recovered by the algorithm. Significant discontinuities are observed along the spanwise direction due to the temporal discontinuity of snapshots between each neighbouring spanwise plane - methods to smoothen the spanwise spurious variations are presented in the section on post-processing techniques. This case-study clearly shows the ability of the algorithm to reconstruct the 3D domain and these results are an improvement on the mirror-imaging technique used by Robinson (2015) for the same data-set in 3D variational data-assimilation. It is also an improvement on the 'empty box' technique where the a numerical simulation, with a superimposed inlet condition at every time-step, is used to create a background condition. Such a methodology is time-consuming and requires high temporal resolution of the data. The SO method is extremely quick $(\sim 8 \mathrm{~s})$ and requires only a long sequence with no limit on the temporal resolution. 


\subsubsection{Wake flow around a circular cylinder at Re 3900}

The PIV experiment was expanded to a more turbulent wake flow in the transitional Reynolds regime at Re of 3900. A similar schematic to the previous experimental setup was used for data generation. A cylinder of diameter $D=12 \mathrm{~mm}$ and length $260 \mathrm{~mm}$ was placed in the air flow. Rectangular end-plates $(100 \mathrm{~mm} \times 85 \mathrm{~mm})$ are fixed $15 \mathrm{~mm}$ from the ends of the cylinder. The free stream velocity $U$ was increased to $5 \mathrm{~m} / \mathrm{s}$ in order to obtained a Reynolds in the range of 3900. On the IP 4108 image pairs are recorded and processed by Davis 10 software from LaVision. In order to account for increased turbulence combined with the noise levels generally associated with PIV data-sets, significantly higher number of snapshots (20 540) were recorded on the OP. The time-difference between two frames for one correlation field was set at $20 \mu \mathrm{s}$ for the IP and at $30 \mu \mathrm{s}$ for the OP. All images were recorded at the highest frequency supported by the camera at $1.617 \mathrm{kHz}$. The OP intersects the IP in the middle at $6.5 \mathrm{D}$ from the centre of the cylinder.

The IP is discretised into $N_{\mathrm{y}} \times N_{\mathrm{z}}=110 \times 92$ mesh-points while the OP is discretised into $N_{\mathrm{x}} \times N_{\mathrm{y}}=69 \times 108$ mesh-points. The issue related to erroneous data at the border of the planes persists and thus the boundary points are excluded in the reconstruction. Thus, only a sub-set of the original snapshot measuring $L_{\mathrm{y}} \times L_{\mathrm{z}}=7 D \times 5.6 D$ on the IP and $L_{\mathrm{x}} \times L_{\mathrm{y}}=4.3 D \times 7 D$ on the OP are extracted. These are discretised into $N_{\mathrm{y}} \times N_{\mathrm{z}}=93 \times 72$ mesh-points for the IP and $N_{\mathrm{x}} \times N_{\mathrm{y}}=64 \times 93$ points for the OP. A sample streamwise velocity field on the IP and the $\mathrm{OP}$ is shown in figure 13
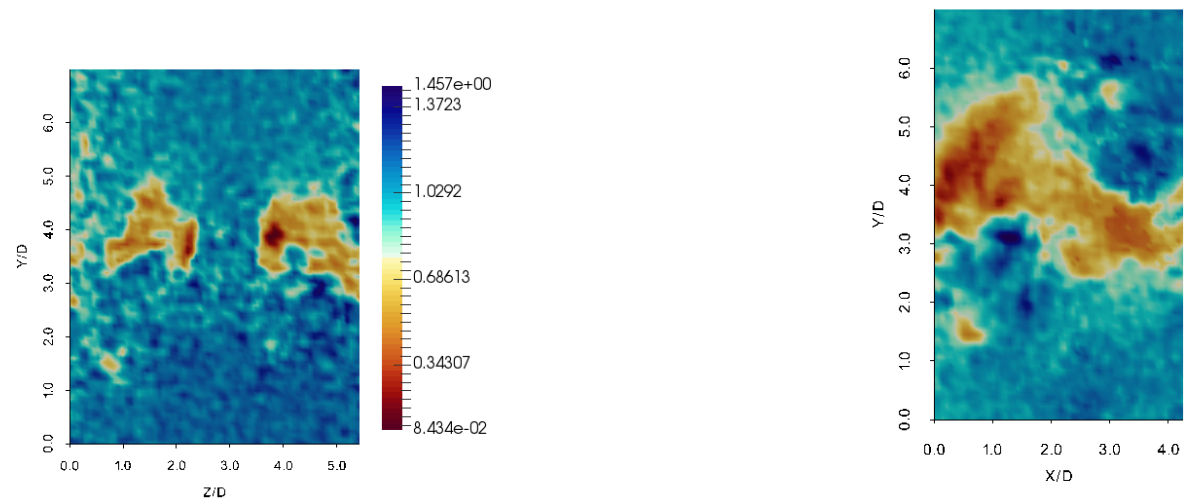

Figure 13: Instantaneous streamwise velocity fields extracted on the IP and the OP from PIV data-set of wake flow around a cylinder at Re of 3900 .

The reconstruction capabilities of the SO method and the averaging SO method are analysed with this flow configuration. The two-way reconstructed velocity fields on the IP are shown in figure 14 along with the reference IP velocity fields. A run-time of $t_{\mathrm{rec}}=9.16 \mathrm{~s}$ was clocked by the $\mathrm{SO}$ algorithm and $t_{\mathrm{rec}}=12.14 \mathrm{~s}$ by the averaging $\mathrm{SO}$ algorithm. Since the experimental data-set is noisy, the SO reconstructed velocity field also contains comparable noise. Thus, spanwise discontinuities, which were clearly visible for all previous cases reconstructed with the SO method, appear attenuated in the reconstruction. Such an attenuation is more a result of the data-deterioration from perfect synthetic or low turbulence data to noisy, highly turbulent data-set than an algorithm improvement. For the averaging SO reconstruction, a dilution of the noise is observed with minimal discontinuities - a slight attenuation of the kinetic energy can be observed with this reconstruction. Both the algorithms manage to reconstruct all the main turbulent structures in the flow as well as capturing significant small-scale structures.

The availability of a long sequence of OP snapshots allows for the study of reconstruction efficiency as a function of OP snapshots. Table 2 tabulates the relative error percentage (in 2D) 
Table 2: Relative error percentage with respect to maximum OP snapshot case (20480) as a function of number of OP snapshots for the experimental data-set of wake flow around a cylinder at Re 3900

\begin{tabular}{lccc}
\hline OP snapshots & Error \%(u) & Error \%(v) & Error \%(w) \\
\hline 2048 & $9.34 \%$ & $16.26 \%$ & $57.86 \%$ \\
4096 & $5.42 \%$ & $6.50 \%$ & $12.38 \%$ \\
6144 & $3.17 \%$ & $5.58 \%$ & $9.47 \%$ \\
8192 & $3.17 \%$ & $5.12 \%$ & $8.09 \%$ \\
10240 & $3.17 \%$ & $3.98 \%$ & $5.48 \%$ \\
12288 & $2.00 \%$ & $3.38 \%$ & $4.97 \%$ \\
14336 & $0.00 \%$ & $2.43 \%$ & $4.55 \%$ \\
16384 & $0.00 \%$ & $1.87 \%$ & $3.72 \%$ \\
18432 & $0.00 \%$ & $0.18 \%$ & $1.11 \%$ \\
20480 & $0.00 \%$ & $0.00 \%$ & $0.00 \%$ \\
\hline
\end{tabular}

between the reconstructed IP and the reference IP as a function of the number of OP snapshots used for the reconstruction. The case associated with maximum OP snapshots of 20480 is used as the base for relative error calculation. The improvement by using 20480 snapshots instead of 2048 is best highlighted in the spanwise component where we obtain a significant $57.86 \%$ improvement. A drastic error reduction is seen for all components by doubling the number of snapshots from 2048 to 4096. An inverse exponential like curve is overall observed with further increase in OP snapshots producing smaller and smaller improvements. By using 14336 snapshots, a 17\% improvement is observed as compared to 2048 snapshots. However, a further increase from 14336 to 20480 produces only 1.5\% improvement on the reconstruction.

These results are promising but in order to adapt the reconstruction methodology to data assimilation studies for creation of 3D backgrounds and/or 3D observations, the volumetric data-set needs to be analysed. Figure 15 plots the $3 \mathrm{D}$ volumetric iso-contours for each velocity component reconstructed from the SO and the averaging SO reconstruction techniques. Due to the $2 \mathrm{D}$ restriction of the experimental data-set, a comparison is not possible, however, large-scale structures can be observed in the iso-contours especially in the averaging SO case. Significant noise reduction in the averaging SO reconstruction is also seen in the iso-contours especially for the spanwise velocity in comparison with the simple case. Figures 14 and 15 are a good indication that meaningful volumetric reconstruction can indeed be obtained using the SO methods for use in data assimilation studies.

\subsubsection{Mixing layer at $\lambda$ of $\mathbf{0 . 3 3}$}

Another stereoscopic PIV experiment was performed in the a plane mixing layer with a modified velocity ratio $\lambda$ of 0.33 where $\lambda=(1-r) /(1+r)$ with $r=U_{\min } / U_{\max }$. A $\lambda$ approaching 0 implies a stable mixing layer while a $\lambda$ approaching 1 implies an unstable mixing layer. The modified velocity ratio was maintained with $U_{\max }=1 \mathrm{~m} / \mathrm{s}$ and $U_{\min }=0.5 \mathrm{~m} / \mathrm{s}$. Both air flows were maintained at the same temperature. The vorticity thickness $\delta_{\omega}$ of the mixing layer at the intersection between IP and OP was estimated to be egual to $51 \mathrm{~mm}$. The Reynolds based on this vorticity thickness and on the velocity difference was equal to 1540 . With a similar imaging setup as the wake flow case 3, 4000 image pairs were acquired at a frequency of $600 \mathrm{~Hz}$. The domain size for the measurement planes were identical to the previous case-study. The OP 

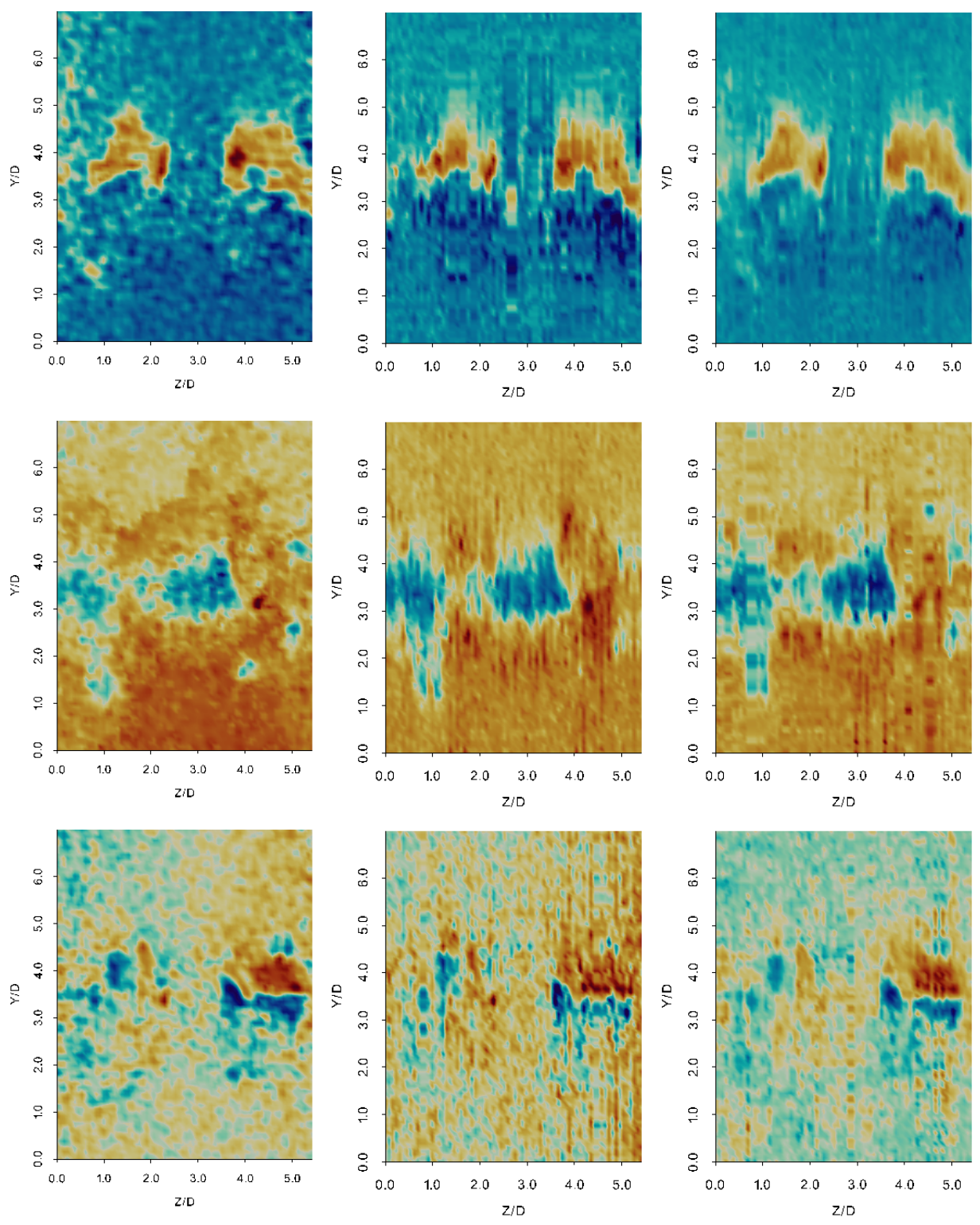

Figure 14: PIV wake flow instantaneous velocity fields extracted on the IP at Re of 3900. From top to bottom: streamwise, vertical and spanwise velocity components. From the left to the right: reference, SO reconstruction, and averaging SO reconstruction.

intersects the IP in the middle and protrudes for $10 \mathrm{~mm}$ behind the IP. The image pairs were analysed by the Davis 7.2 software from LaVision providing 4000 vector fields for both OP and IP. This PIV data-set was consistant with hot-wire measurements carried out by Sodjavi \& Carlier (2013) in the same wind tunnel.

The IP is discretised into $N_{\mathrm{y}} \times N_{\mathrm{z}}=175 \times 134$ mesh-points and the OP into $N_{\mathrm{x}} \times N_{\mathrm{y}}=140 \times 174$ mesh-points. Ignoring the borders and the region far away from the mixing layer, a subset of the IP measuring $L_{\mathrm{y}} \times L_{\mathrm{z}}=120 \mathrm{~mm} \times 138 \mathrm{~mm}$ and $L_{\mathrm{x}} \times L_{\mathrm{y}}=122 \mathrm{~mm} \times 120 \mathrm{~mm}$ for the OP was extracted. This sub-set contains $N_{\mathrm{x}} \times N_{\mathrm{y}}=100 \times 100$ points in the OP and $N_{\mathrm{y}} \times N_{\mathrm{z}}=100 \times 115$ points in the IP. The sample streamwise velocity field on this sub-set is provided in figure 16

Figure 17 depicts the original and reconstructed velocity fields on the inlet. The SO method required $t_{\text {rec }}=6.85 \mathrm{~s}$ to recover the velocity field for this case-study. All three components are reconstructed quite well by the algorithm. One can see that the interface between the 

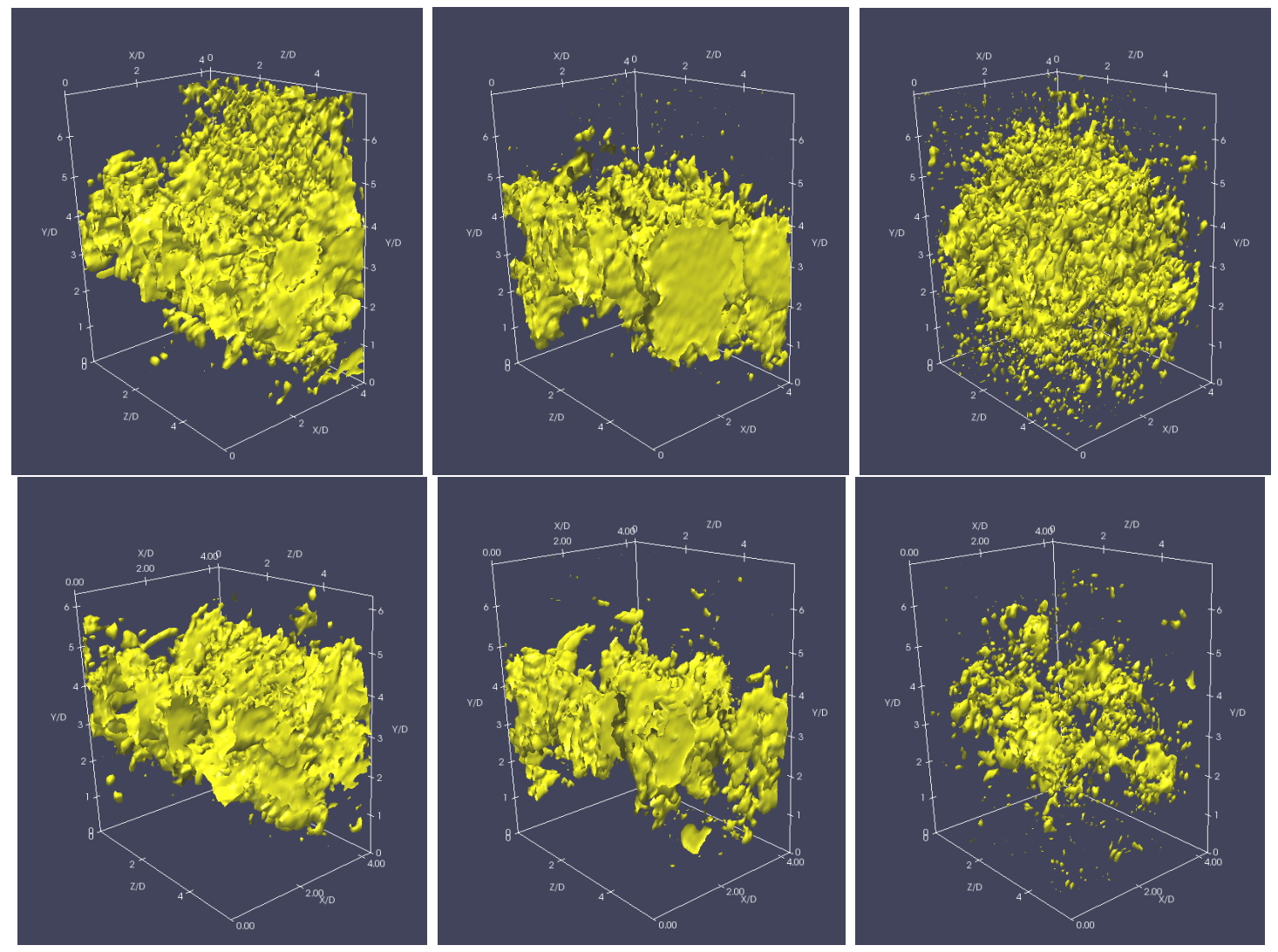

Figure 15: PIV wake flow instantaneous velocity iso-contours extracted on the 3D domain. From top to bottom: SO reconstruction and averaging SO reconstruction. From the left to the right: streamwise, vertical and spanwise velocity components.
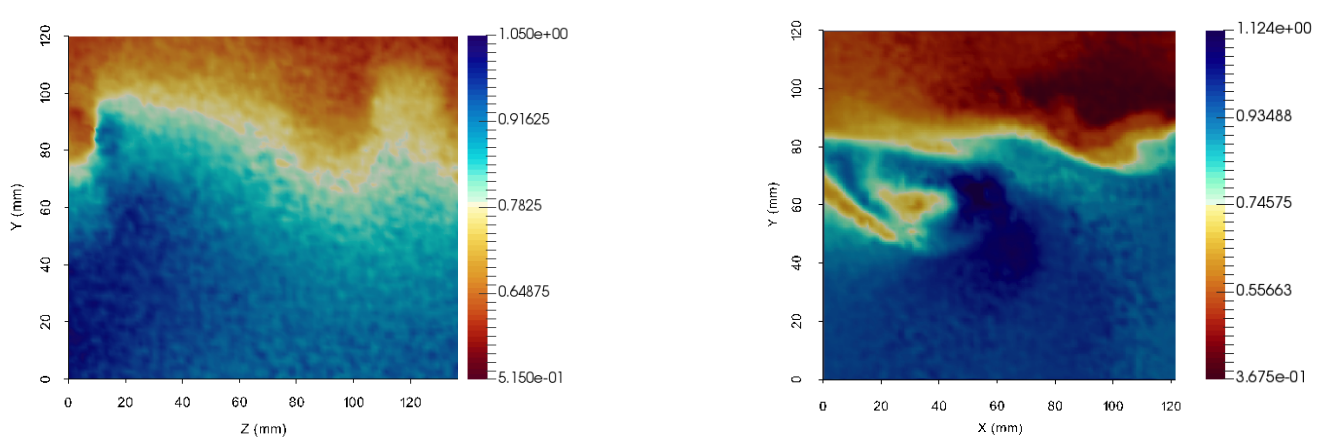

Figure 16: Instantaneous streamwise velocity fields extracted on the IP and the OP from PIVs data-set of the mixing layer.

two fields is very accurately reconstructed by the SO method. For the vertical and spanwise components, a noisy but well-represented reconstruction is obtained. It is interesting to note here that the amount of discontinuities is quite less as compared to previous reconstructions due to smoothness of the reference velocity.

These case studies clearly indicate the ability of the $\mathrm{SO}$ algorithm to reconstruct the flow using 2D planar data. An important advantage of this reconstruction method is the time taken which is in the order of seconds. Most DA methods and other complex reconstruction algorithms take much longer to provide results. 

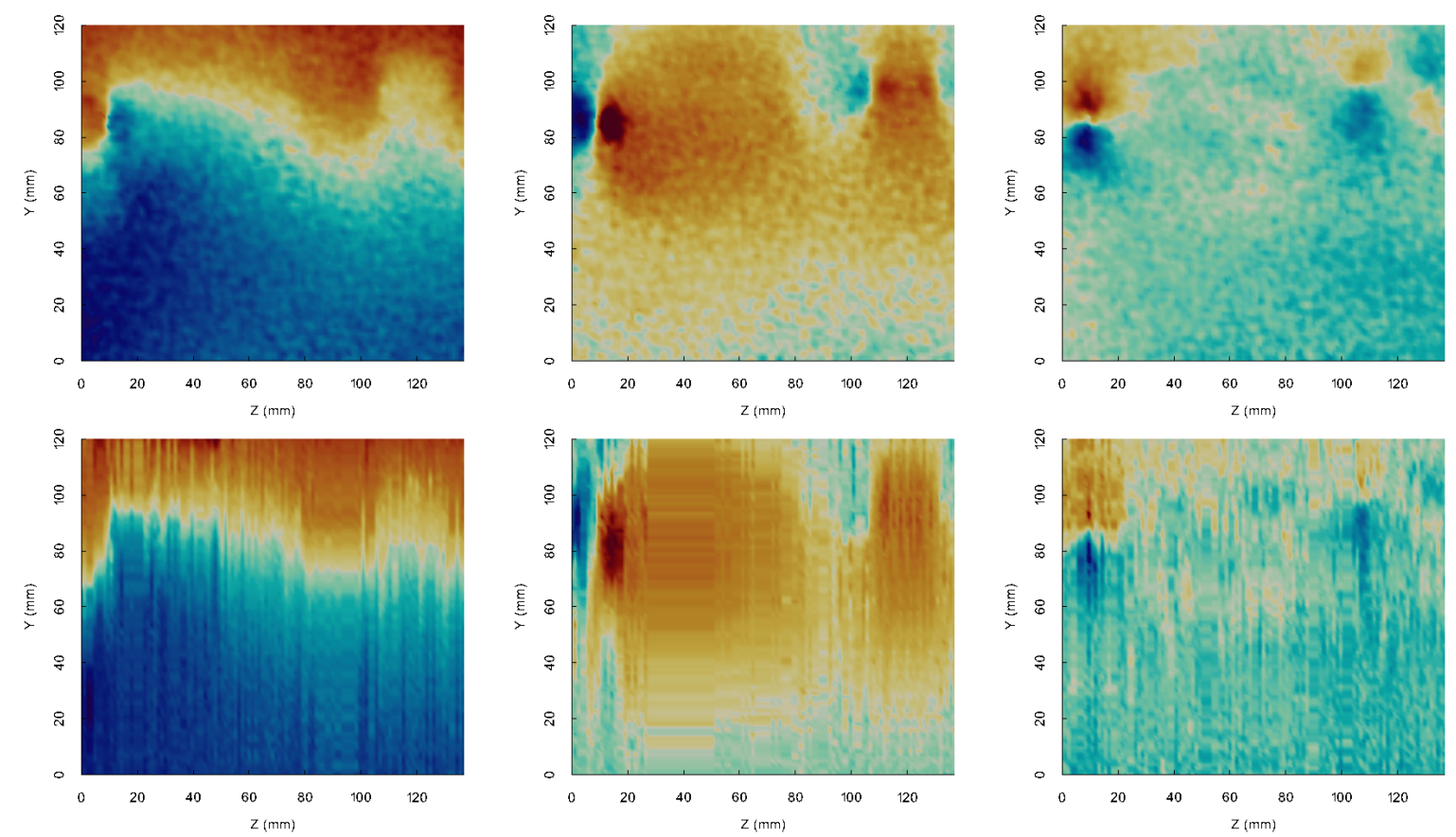

Figure 17: PIV mixing layer instantaneous velocity fields extracted on the IP. From top to bottom: reference and reconstruction. From the left to the right: streamwise, vertical and spanwise velocity components.

\subsection{Error characterisation}

This final section on error characteristics analyses quantitatively the performance of the SO reconstruction algorithm with respect each of the cases considered above. The accuracy of the reconstruction is quantified through a root mean square error (RMSE) comparison between the reconstruction and the true reference for each of the three velocity components. For the synthetic case of wake flow around a circular cylinder (case1) and channel flow (case2) the RMSE is calculated over the whole volumetric domain on which we have the reference readily available. For the experimental case studies: wake flow at Re 300 (case3), at Re 3900 (case4), and mixing layer (case5), the RMSE is calculated only on the IP on which we have the reference data. The corresponding RMSE errors are plotted in figure 18 .

The error plot provides some valuable insights in to the performance of the model:

- For case3 with the wake flow at Re 300, the error is least due to the organised laminar nature of the flow. Clearly, the algorithm is able to better represent the flow when the level of turbulence is low.

- Considering the RMSE for case2 - channel flow synthetic DNS, the low error in comparison to the other flows reiterates the previous point where in homogenous turbulence, as in channel flow, is more easily and better reconstructed using the SO algorithm. In addition, for the case of homogenous turbulence with high resolution data, using additional OP data-set (as with the 1 plane (1p), 5 planes (5p), and 10 planes (10p) cases) does not provide a significant improvement of the reconstruction. This is due to the limitation of the algorithm towards optimising the small scales of turbulence which predominate in channel flow especially with high resolution data-sets.

- The averaging SO methods (ASO) provides, in all considered cases, an improvement on the simple SO method. 


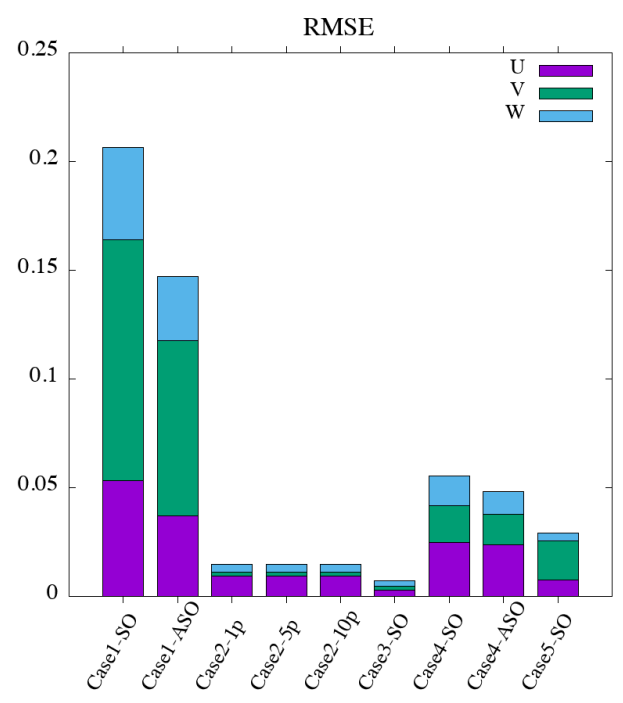

Figure 18: Root mean square error for each velocity component between the reconstruction and the reference for all case studies. For cases where the averaging SO method (ASO) has been analysed, the corresponding error is also provided. For cases with 3D reference, the RMSE is calculated in the volume while for the rest it is calculated on the 2D IP. Legend: Case1 synthetic wake flow at Re 3900; Case2 - synthetic channel flow at $\operatorname{Re}_{\tau}$ 590; Case3 - PIV wake flow at Re 300; Case4 - PIV wake flow at Re 3900; Case5 - PIV mixing layer.

- The comparatively large RMSE obtained for case1 is due to the calculation performed over the volume where in the error is expected to be higher than on the IP.

\section{Conclusion}

This present study investigated a novel flow reconstruction method to obtain 3D volumetric velocity measurements using $2 \mathrm{D}$ planar measurements. The reconstruction algorithm is based on the assumption that considering the periodicity of the flow perpendicular to the $\mathrm{OP}$, a sufficiently long time-sequence of snapshots on the OP contains at some instance within that time-sequence a representation of each of the parallel $2 \mathrm{D}$ planes comprising the $3 \mathrm{D}$ volumetric domain encompassed by the OP and the IP. Three versions of the minimisation algorithm were formulated and tested on two synthetic case studies and three experimental case studies. The algorithm is shown to reconstruct the main turbulent structures of the flow for all cases. Good performance is shown for the three wake flow studies while for the mixing layer, small scale structures are absent in the reconstruction. For a higher-dimension system such as the channel DNS data-set, the algorithm shows significant discontinuities while marginal oscillations are found in the other cases. Methods for addressing these issues are also analysed, and applied to the synthetic wake flow, showing significant reduction when POD based SO method or Averaging SO methods are employed - the 3D turbulent structures are shown to match well with the reference field. The averaging SO method, which provided the best reconstruction for the synthetic case, was also applied to the experimental data-set on wake flow at Re of 3900. The method was shown to reduce noise and smooth the reconstructed velocity. The volumetric iso-contours showed meaningful large-scale structures are reconstructed using this algorithm. Techniques to ensure a divergence free reconstruction are also explored. Given the successful application of the SO methodology, especially with PIV sparse data-sets, it could be an useful tool for performing variational DA studies where in an accurate background, constructed using the SO method, can help converge to the optimal solution with less iterations 
and thus lower cost. In addition, the use of the POD based SO can help calculate the so called background covariance matrix for variational DA which is an important weighting factor in the cost functional. The algorithm can also be expanded to reconstruct axisymmetric flows such as flow through a pipe or jet flows.

\section{Acknowledgements}

The authors would like to thank Anthony Guibert for providing the experimental data-sets (cases 3 and 5), and Sylvain Laizet for the channel flow DNS data-set.

\section{References}

Adrian, R. J. 1984 Scattering particle characteristics and their effect on pulsed laser measurements of fluid flow: speckle velocimetry vs particle image velocimetry. Appl. Opt. 23 (11), $1690-1691$.

Braud, C., Heitz, D., Braud, P., Arroyo, G. \& Delville, J. 2004 Analysis of the wake mixing layer interaction using multiple plane PIV and 3d classical POD. Experiments in fluids 37 (1), 95-104.

Brücker, C. 1995 Digital-particle-image-velocimetry (DPIV) in a scanning light-sheet: 3d starting flow around a short cylinder. Experiments in Fluids 19 (4), 255-263.

Brücker, C., Hess, D. \& Kitzhofer, J. 2012 Single-view volumetric PIV via high-resolution scanning, isotropic voxel restructuring and 3d least-squares matching (3d-LSM). Measurement Science and Technology 24 (2), 024001.

Casey, T. A., Sakakibara, J. \& Thoroddsen, S. T. 2013 Scanning tomographic particle image velocimetry applied to a turbulent jet. Physics of fluids 25 (2), 025102.

Chandramouli, P., Memin, E. \& Heitz, D. 2017 4d Turbulent Wake Reconstruction using Large Eddy Simulation based Variational Data Assimilation. Delft, Netherlands.

Chandramouli, P., Memin, E., Heitz, D. \& Laizet, S. 2018 Coarse large-eddy simulations in a transitional wake flow with flow models under location uncertainty. Comput. Fluids 168, 170-189.

D'adamo, J., Papadakis, N., Mémin, E. \& Artana, G. 2007 Variational assimilation of POD low-order dynamical systems. J. Turbul. 8, N9.

Foucaut, J.-M.and Coudert, S., Stanislas, M. \& Delville, J. 2011 Full 3d correlation tensor computed from double field stereoscopic PIV in a high Reynolds number turbulent boundary layer. Experiments in Fluids $\mathbf{5 0}$ (4), 839-846.

Fujisawa, N., Tanahashi, S. \& Srinivas, K. 2005 Evaluation of pressure field and fluid forces on a circular cylinder with and without rotational oscillation using velocity data from PIV measurement. Meas. Sci. Technol. 16 (4), 989-996.

Ganapathisubramani, B., Lakshminarasimhan, K. \& Clemens, N. T. 2008 Investigation of three-dimensional structure of fine scales in a turbulent jet by using cinematographic stereoscopic particle image velocimetry. Journal of fluid mechanics 598, 141-175.

Gesemann, S., Huhn, F., Schanz, D. \& Schröder, A. 2016 From noisy particle tracks to velocity, acceleration and pressure fields using B-splines and penalties. In 18th international symposium on applications of laser and imaging techniques to fluid mechanics, Lisbon, Portugal, pp. $4-7$. 
Gronskis, A., Heitz, D. \& Mémin, E. 2013 Inflow and initial conditions for direct numerical simulation based on adjoint data assimilation. J. Comput. Phys. 242, 480-497.

Hamdi, J., Assoum, H., Abed-Meraïm, K. \& Sakout, A. 2018 Volume reconstruction of an impinging jet obtained from stereoscopic-PIV data using POD. European Journal of MechanicsB/Fluids 67, 433-445.

Kähler, C. J. \& Kompenhans, J. 2000 Fundamentals of multiple plane stereo particle image velocimetry. Experiments in Fluids 29 (1), S070-S077.

Kit, E., Krivonosova, O., Zhilenko, D. \& Friedman, D. 2005 Reconstruction of large coherent structures from SPIV measurements in a forced turbulent mixing layer. Exp. Fluids 39 (4), $761-770$.

Laizet, S. \& Lamballais, E. 2009 High-order compact schemes for incompressible flows: A simple and efficient method with quasi-spectral accuracy. J. Comput. Phys. 228 (16), 5989-6015.

Laizet, S. \& Li, N. 2011 Incompact3d: A powerful tool to tackle turbulence problems with up to $\mathrm{O}(105)$ computational cores. Int. J. Numer. Methods Fluids 67 (11), 1735-1757.

Le Dimet, F.-X. \& Talagrand, O. 1986 Variational algorithms for analysis and assimilation of meteorological observations: theoretical aspects. Tellus A 38 (2), 97-110.

Lions, J. 1971 Optimal control of systems governed by partial differential equations problèmes aux limites. Berlin: Springer p. 407.

Mons, V., Chassaing, J.-C., Gomez, T. \& Sagaut, P. 2016 Reconstruction of unsteady viscous flows using data assimilation schemes. J. Comput. Phys. 316, 255-280.

Robinson, C. 2015 Image assimilation techniques for Large Eddy Scale models : Application to 3d reconstruction. Scientific, Université de Rennes 1, Rennes.

Scarano, F. 2012 Tomographic PIV: principles and practice. Measurement Science and Technology 24 (1), 012001.

Schanz, D., Gesemann, S. \& Schröder, A. 2016 Shake-The-Box: Lagrangian particle tracking at high particle image densities. Experiments in Fluids 57 (5).

Schneiders, J. F. G. \& Scarano, F. 2016 Dense velocity reconstruction from tomographic PTV with material derivatives. Exp. Fluids $\mathbf{5 7}$ (9).

de Silva, C. M., Philip, J. \& Marusic, I. 2013 Minimization of divergence error in volumetric velocity measurements and implications for turbulence statistics. Experiments in Fluids $54(7)$.

Sodjavi, K. \& Carlier, J. 2013 Experimental study of thermal mixing layer using variable temperature hot-wire anemometry. Exp. Fluids 54 (10).

Stansby, P. K. 1974 The effects of end plates on the base pressure coefficient of a circular cylinder. Aeronaut. J. 78 (757), 36-37.

Steinberg, A. M., Driscoll, J. F. \& Ceccio, S. L. 2009 Three-dimensional temporally resolved measurements of turbulenceflame interactions using orthogonal-plane cinema-stereoscopic PIV. Experiments in fluids 47 (3), 527-547.

Van Bladel, J. 1958 On Helmholtz's Theorem in Finite Regions. IRE Transaction on Antennas and Propagation . 
Wang, C., Gao, Q., Wang, H., Wei, R., Li, T. \& Wang, J. 2016 Divergence-free smoothing for volumetric PIV data. Experiments in Fluids $\mathbf{5 7}$ (1).

Wang, H., Gao, Q., Wang, S., Li, Y., Wang, Z. \& Wang, J. 2018 Error reduction for timeresolved PIV data based on NavierStokes equations. Experiments in Fluids 59 (10).

Yang, Y., Robinson, C., Heitz, D. \& Mémin, E. 2015 Enhanced ensemble-based 4dvar scheme for data assimilation. Comput. Fluids 115, 201-210.

Zhang, W., Hain, R. \& Kähler, C. J. 2008 Scanning PIV investigation of the laminar separation bubble on a SD7003 airfoil. Experiments in Fluids 45 (4), 725-743. 